\title{
QUANTIZATION FOR A MIXTURE OF UNIFORM DISTRIBUTIONS ASSOCIATED WITH PROBABILITY VECTORS
}

\author{
Mrinal Kanti Roychowdhury — Wasiela Salinas \\ School of Mathematical and Statistical Sciences, University of Texas Rio Grande Valley, USA
}

\begin{abstract}
The basic goal of quantization for probability distribution is to reduce the number of values, which is typically uncountable, describing a probability distribution to some finite set and thus approximation of a continuous probability distribution by a discrete distribution. Mixtures of probability distributions, also known as mixed distributions, are an exciting new area for optimal quantization. In this paper, we investigate the optimal quantization for three different mixed distributions generated by uniform distributions associated with probability vectors.
\end{abstract}

Communicated by Manfred Kühleitner

\section{Introduction}

Continuous-valued signals can take any real value either in the entire range of real numbers or in a range limited by some system constraints. In either of the two cases, an uncountably infinite set of values is required to represent the signal values. If a signal has to be processed or stored digitally, each of its values must be representable by a finite number of bits. Thus, all values together have to form a finite countable set. A signal consisting only of such discrete values is said to be quantized. The process of transformation of a continuous-valued signal into a discrete-valued one is called 'quantization'. It has broad application in engineering and technology (see GG, GN, Z). For mathematical treatment

(C) 2020 BOKU-University of Natural Resources and Life Sciences and Mathematical Institute, Slovak Academy of Sciences.

2010 Mathematics Subject Classification: 60Exx, 94A34.

Keyw ords: Mixed distribution, uniform distribution, optimal sets, quantization error, quantization dimension, quantization coefficient.

Licensed under the Creative Commons Attribution-NC-ND 4.0 International Public License. 
of quantization one is referred to Graf-Luschgy's book (see [GL1]). Let $\mathbb{R}^{d}$ denote the $d$-dimensional Euclidean space equipped with the Euclidean norm $\|\cdot\|$, and let $P$ be a Borel probability measure on $\mathbb{R}^{d}$. Then, the $n$th quantization error for $P$, with respect to the squared Euclidean distance, is defined by

$$
\begin{aligned}
V_{n}:=V_{n}(P)=\inf \{V(P ; \alpha): \alpha & \left.\subset \mathbb{R}^{d}, \operatorname{card}(\alpha) \leq n\right\}, \\
& \text { where } V(P ; \alpha):=\int \min _{a \in \alpha}\|x-a\|^{2} \mathrm{~d} P(x)
\end{aligned}
$$

represents the distortion error for $P$ due to the set $\alpha$. A set $\alpha \subset \mathbb{R}^{d}$ is called an optimal set of $n$-means for $P$ if $V_{n}(P)=V(P$; $\alpha)$. It is known that for a continuous Borel probability measure an optimal set of $n$-means always has exactly $n$-elements (see GL1]). Optimal sets of $n$-means for different probability distributions were calculated by several authors, for example, one can see CR, DR1, DR2, GL2, RR1, L1, R1 R5. The number

$$
\lim _{n \rightarrow \infty} \frac{2 \log n}{-\log V_{n}(P)}
$$

if it exists, is called the quantization dimension of the probability measure $P$, and is denoted by $D(P)$; on the other hand, for any $s \in(0,+\infty)$, the number $\lim _{n \rightarrow \infty} n^{\frac{2}{s}} V_{n}(P)$, if it exists, is called the $s$-dimensional quantization coefficient for $P$ (see [GL1, P]).

Let us now state the following proposition (see [GG, GL1]):

Proposition 1.1. Let $\alpha$ be an optimal set of n-means for $P$, and $a \in \alpha$. Then, (i) $P(M(a \mid \alpha))>0$, (ii) $P(\partial M(a \mid \alpha))=0$, (iii) $a=E(X: X \in M(a \mid \alpha))$, where $M(a \mid \alpha)$ is the Voronoi region of $a \in \alpha$, i.e., $M(a \mid \alpha)$ is the set of all elements $x$ in $\mathbb{R}^{d}$ which are closest to a among all the elements in $\alpha$.

Proposition 1.1 says that if $\alpha$ is an optimal set and $a \in \alpha$, then $a$ is the conditional expectation of the random variable $X$ given that $X$ takes values in the Voronoi region of $a$. The following theorem is known.

TheOrem 1.2 (see RR2]). Let $P$ be a uniform distribution on the closed interval $[a, b]$. Then, the optimal set $n$-means is given by $\alpha_{n}:=\left\{a+\frac{2 i-1}{2 n}(b-a)\right.$ : $1 \leq i \leq n\}$, and the corresponding quantization error is $V_{n}:=V_{n}(P)=\frac{(a-b)^{2}}{12 n^{2}}$.

THEOREM 1.3. Let $\alpha_{n}$ be an optimal set of n-means for a uniform distribution on the unit circular arc $S$ given by

$$
S:=\{(\cos \theta, \sin \theta): \alpha \leq \theta \leq \beta\} \text {, where } 0 \leq \alpha<\beta \leq 2 \pi .
$$


Then,

$$
\begin{aligned}
\alpha_{n}:=\left\{\frac{2 n}{\beta-\alpha} \sin \left(\frac{\beta-\alpha}{2 n}\right)(\right. & \cos \left(\alpha+(2 j-1) \frac{\beta-\alpha}{2 n}\right), \\
& \left.\left.\sin \left(\alpha+(2 j-1) \frac{\beta-\alpha}{2 n}\right)\right): j=1,2, \ldots, n\right\}
\end{aligned}
$$

forms an optimal set of $n$-means, and the corresponding quantization error is given by

$$
V_{n}=\frac{(\alpha-\beta)^{2}-2 n^{2}+2 n^{2} \cos \frac{\alpha-\beta}{n}}{(\alpha-\beta)^{2}} .
$$

Proof. Notice that $S$ is an arc of the unit circle $x_{1}^{2}+x_{2}^{2}=1$ which subtends a central angle of $\beta-\alpha$ radian, and the probability distribution is uniform on $S$. Hence, the density function is given by $f\left(x_{1}, x_{2}\right)=\frac{1}{\beta-\alpha}$ if $\left(x_{1}, x_{2}\right) \in S$, and zero, otherwise. Thus, the proof follows in the similar way as the proof in the similar theorem in [RR2].

Mixed distributions are an exciting new area for optimal quantization. For any two Borel probability measures $P_{1}$ and $P_{2}$, and $p \in(0,1)$, if $P:=p P_{1}+(1-p) P_{2}$, then the probability measure $P$ is called the mixture or the mixed distribution generated by the probability measures $\left(P_{1}, P_{2}\right)$ associated with the probability vector $(p, 1-p)$. Such kind of problems has rigorous applications in many areas including signal processing. For example, while driving long distances, we have seen sometimes cellular signals get cut off. This happens because of being far away from the tower, or there is no tower nearby to catch the signal. In optimal quantization for mixed distributions one of our goals is to find the exact locations of the towers by giving different weights, also called importance, to different portions of a path.

The following theorem about the quantization dimension for the mixed distributions is well-known. For some more details please see [L, Theorem 2.1].

Theorem 1.4. Let $P_{1}$ and $P_{2}$ be any two Borel probability measures on $\mathbb{R}^{d}$ such that both $D\left(P_{1}\right)$ and $D\left(P_{2}\right)$ exist. If $P=p P_{1}+(1-p) P_{2}$, where $0<p<1$, then $D(P)=\max \left\{D\left(P_{1}\right), D\left(P_{2}\right)\right\}$.

In this paper, in Section 2, we have considered a mixed distribution generated by two uniform distributions on a circle and on one of its diameters associated with the probability vector $\left(\frac{1}{2}, \frac{1}{2}\right)$. For this mixed distribution, in Theorem 2.10. we have explicitly determined the optimal sets of $n$-means and the $n$th quantization errors for all positive integers $n \geq 2$. In Proposition 2.12, we have proved that the quantization dimension $D(P)$ of the mixed distribution is one, which 
supports Theorem 1.4 because $D\left(P_{1}\right)=D\left(P_{2}\right)=1$, and the quantization coefficient exists as a finite positive number which equals $\frac{3}{8}\left(4+\pi^{2}\right)$. Optimal sets of $n$-means and the $n$th quantization errors are calculated, in Section 3, for the mixture of two uniform distributions on two disconnected line segments $\left[0, \frac{1}{2}\right]$ and $\left[\frac{3}{4}, 1\right]$ associated with the probability vector $\left(\frac{3}{4}, \frac{1}{4}\right)$, and in Section 4 , for the mixture of two uniform distributions on two connected line segments $\left[0, \frac{1}{2}\right]$ and $\left[\frac{1}{2}, 1\right]$ associated with the probability vector $\left(\frac{3}{4}, \frac{1}{4}\right)$. We would like to mention that in these two sections, to determine the optimal sets of $n$-means and the $n$th quantization errors for the mixed distributions we need to take the help of two different sequences $\{a(n)\}_{n=1}^{\infty}$ given by Definition 3.8, and Definition 4.6. If the probability vector $\left(\frac{3}{4}, \frac{1}{4}\right)$ is replaced by some other probability vector $(p, 1-p)$, where $0<p<1$, what will be the two such sequences are not known yet. In fact, optimal sets of $n$-means and the $n$th quantization errors are not known yet for a more general mixed distribution.

\section{Quantization for a mixed distribution on the circles including a diameter}

Let $i$ and $j$ be the unit vectors in the positive directions of the $x_{1}$ - and $x_{2}$-axes, respectively. By the position vector $a$ of a point $A$, it is meant that $\overrightarrow{O A}=a$. We will identify the position vector of a point $\left(a_{1}, a_{2}\right)$ by $\left(a_{1}, a_{2}\right):=$ $a_{1} i+a_{2} j$, and apologize for any abuse in notation. For any two position vectors $a:=\left(a_{1}, a_{2}\right)$ and $b:=\left(b_{1}, b_{2}\right)$, we write $\rho(a, b):=\left\|\left(a_{1}, b_{1}\right)-\left(a_{2}, b_{2}\right)\right\|^{2}=\left(a_{1}-\right.$ $\left.a_{2}\right)^{2}+\left(b_{1}-b_{2}\right)^{2}$, which gives the squared Euclidean distance between the two points $\left(a_{1}, a_{2}\right)$ and $\left(b_{1}, b_{2}\right)$. Let $P$ and $Q$ belong to an optimal set of $n$-means for some positive integer $n$, and let $D$ be a point on the boundary of the Voronoi regions of the points $P$ and $Q$. Since the boundary of the Voronoi regions of any two points is the perpendicular bisector of the line segment joining the points, we have $|\overrightarrow{D P}|=|\overrightarrow{D Q}|$, i.e., $(\overrightarrow{D P})^{2}=(\overrightarrow{D Q})^{2}$ implying $(p-d)^{2}=(q-d)^{2}$, i.e., $\rho(d, p)-\rho(d, q)=0$. We call such an equation a canonical equation. By $E(X)$ and $V:=V(X)$, we represent the expectation and the variance of a random variable $X$ with respect to the probability distribution under consideration.

Let $P_{1}$ be the uniform distribution defined on the circle $x_{1}^{2}+x_{2}^{2}=1$ with center $O(0,0)$, and $P_{2}$ be the uniform distribution on one of its diameters. Let us denote the diameter by $L_{1}$ and the circle by $L_{2}$. Without any loss of generality, we can assume that the diameter is horizontal, i.e., the diameter is represented by

$$
L_{1}:=\left\{\left(x_{1}, 0\right):-1 \leq x_{1} \leq 1\right\}
$$




\section{QUANTIZATION FOR A MIXTURE OF UNIFORM DISTRIBUTIONS}

which intersects the circle at the two points $A(-1,0)$ and $B(0,1)$. Let $L$ be the path formed by the circle and the diameter $A B$. Thus, we have

where

$$
L=L_{1} \cup L_{2},
$$

$$
L_{1}=\{(t, 0):-1 \leq t \leq 1\}, \quad \text { and } \quad L_{2}=\{(\cos \theta, \sin \theta): 0 \leq \theta \leq 2 \pi\} .
$$

Let $s$ represent the distance of any point on $L$ from the origin tracing along the boundary $L$ in the positive direction of the $x_{1}$-axis, and in the counterclockwise direction. Thus, $s=1$ represents the point $B(1,0), s=1+\frac{\pi}{2}$ represents the point $(0,-1)$, and so on. Take the mixed distribution $P$ as

$$
P:=\frac{1}{2} P_{1}+\frac{1}{2} P_{2},
$$

i.e., $P$ is generated by $\left(P_{1}, P_{2}\right)$ associated with the probability vector $\left(\frac{1}{2}, \frac{1}{2}\right)$. For this mixed distribution $P$ in this section, we determine the optimal sets of $n$-means and the $n$th quantization errors for all $n \in \mathbb{N}$. The probability density function (pdf) $f\left(x_{1}, x_{2}\right)$ for the mixed distribution $P$ is given by

$$
f\left(x_{1}, x_{2}\right)= \begin{cases}\frac{1}{4} & \text { if }\left(x_{1}, x_{2}\right) \in L_{1}, \\ \frac{1}{4 \pi} & \text { if }\left(x_{1}, x_{2}\right) \in L_{2} .\end{cases}
$$

On $L_{1}$ we have $\mathrm{d} s=\sqrt{\left(\frac{\mathrm{d} x_{1}}{\mathrm{~d} t}\right)^{2}+\left(\frac{\mathrm{d} x_{2}}{\mathrm{~d} t}\right)^{2}} \mathrm{~d} t=\mathrm{d} t$ yielding $\mathrm{d} P(s)=P(\mathrm{~d} s)=$ $f\left(x_{1}, x_{2}\right) \mathrm{d} s=\frac{1}{4} \mathrm{~d} t$. Similarly, on $L_{2}$, we have $\mathrm{d} s=\mathrm{d} \theta$ yielding $\mathrm{d} P(s)=$ $P(\mathrm{~d} s)=f\left(x_{1}, x_{2}\right) \mathrm{d} s=\frac{1}{4 \pi} \mathrm{d} \theta$.

LEMмa 2.1. Let $X$ be a continuous random variable with mixed distribution taking values on $L$. Then,

$$
E(X)=(0,0) \quad \text { and } \quad V:=V(X)=\frac{2}{3} .
$$

P r o of. We have,

$$
E(X)=\int_{L}\left(x_{1} i+x_{2} j\right) \mathrm{d} P=\frac{1}{4} \int_{L_{1}}(t, 0) \mathrm{d} t+\frac{1}{4 \pi} \int_{L_{2}}(\cos \theta, \sin \theta) \mathrm{d} \theta=(0,0) .
$$

To calculate the variance, we know that $V(X)=E\|X-E(X)\|^{2}$, which implies

$$
V(X)=\frac{1}{4} \int_{L_{1}} \rho((t, 0),(0,0)) \mathrm{d} t+\frac{1}{4 \pi} \int_{L_{2}} \rho((\cos \theta, \sin \theta),(0,0)) \mathrm{d} \theta=\frac{2}{3} .
$$

Thus, the lemma is yielded. 


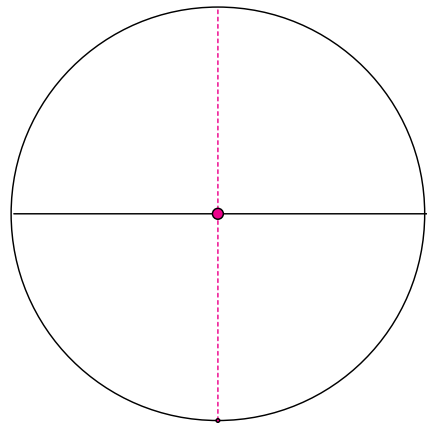

(i)

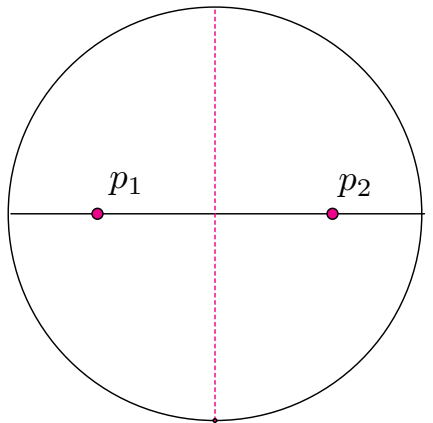

(ii)

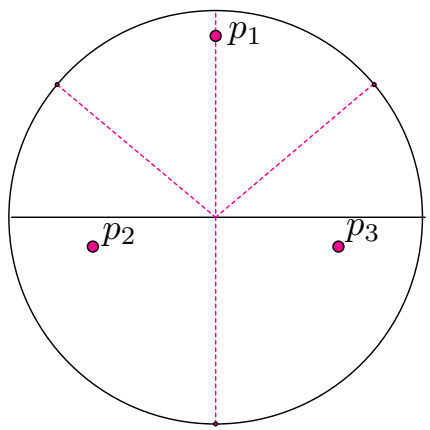

(iii)

Figure 1.

REMARK 2.2. Using the standard theory of probability, for any $(a, b) \in \mathbb{R}^{2}$, we have

$$
E\|X-(a, b)\|^{2}=\int_{L}\left\|\left(x_{1}, x_{2}\right)-(a, b)\right\|^{2} \mathrm{~d} P=V(X)+\|(a, b)-(0,0)\|^{2},
$$

which is minimum if $(a, b)=(0,0)$, and the minimum value is $V(X)$. Thus, we see that the optimal set of one-mean is the set $\{(0,0)\}$, and the corresponding quantization error is the variance $V:=V(X)$ of the random variable $X$ (see Figure1 (i)).

Proposition 2.3. The set $\left\{\left(-\frac{1}{4}-\frac{1}{\pi}, 0\right),\left(\frac{1}{4}+\frac{1}{\pi}, 0\right)\right\}$ forms the optimal set of two-means, and the corresponding quantization error is given by $V_{2}=0.343691$.

P r o of. Since $P$ is a mixed distribution giving the equal weights to both the component probabilities $P_{1}$ and $P_{2}$, and the path $L$ is symmetric with respect to the $x_{2}$-axis, without going into much calculation, we can assume that the 


\section{QUANTIZATION FOR A MIXTURE OF UNIFORM DISTRIBUTIONS}

boundary of the Voronoi regions of the two points in an optimal set of two-means lies along the $x_{2}$-axis. Thus, the optimal set of two-means is given by $\left\{p_{1}, p_{2}\right\}$ (see Figure 1 (ii)), where

$$
\begin{aligned}
p_{1} & =E(X: X \in \overline{A O} \cup \quad \text { (left half of the circle) }) \\
& =\frac{\frac{1}{4} \int_{-1}^{0}(x, 0) \mathrm{d} x+\frac{1}{4 \pi} \int_{\frac{\pi}{2}}^{\frac{3 \pi}{2}}(\cos \theta, \sin \theta) \mathrm{d} \theta}{\frac{1}{4} \int_{-1}^{0} \mathrm{~d} x+\frac{1}{4 \pi} \int_{\frac{\pi}{2}}^{\frac{3 \pi}{2}} \mathrm{~d} \theta} \\
& =\left(-\frac{1}{4}-\frac{1}{\pi}, 0\right),
\end{aligned}
$$

and similarly, $p_{2}=\left(\frac{1}{4}+\frac{1}{\pi}, 0\right)$. The quantization error for two-means is given by

$$
V_{2}=2\left(\frac{1}{4} \int_{-1}^{0} \rho\left((x, 0), p_{1}\right) \mathrm{d} x+\frac{1}{4 \pi} \int_{\frac{\pi}{2}}^{\frac{3 \pi}{2}} \rho\left((\cos \theta, \sin \theta), p_{1}\right) \mathrm{d} \theta\right)=0.343691 .
$$

Thus, the proposition is yielded.

The following proposition gives the optimal set of three-means (see Figure 1 (iii)). The proof follows in the similar way as Proposition 2.5] which is given later.

Proposition 2.4. The set

$$
\{(0,0.877439),(-0.593906,-0.14179),(0.593906,-0.14179)\}
$$

forms an optimal set of three-means, and the corresponding quantization error is given by $V_{3}=0.2386$.

Proposition 2.5. The set

$$
\{(0,0.90407),(-0.633881,0),(0,-0.90407),(0.633881,0)\}
$$

forms an optimal set of four-means, and the corresponding quantization error is given by $V_{4}=0.163013$.

P r o of. Let $\alpha:=\left\{p_{1}, p_{2}, p_{3}, p_{4}\right\}$ be an optimal set of four-means. The following cases can arise:

Case 1. $\alpha$ contains one point from $L_{1}$, the Voronoi region of which does not contain any point from $L_{2}$.

In this case, we can assume that $p_{1}, p_{2}, p_{3}, p_{4}$ can be located as shown in Figure 2(i). Let the boundary of the Voronoi regions of $p_{1}$ and $p_{2}$ intersect $L_{2}$ at the point $d_{1}$ given by the parametric value $\theta=\pi-b$, where $0<b<\frac{\pi}{2}$, and 
the boundary of the Voronoi regions of $p_{2}$ and $p_{3}$ intersect $L_{1}$ at the point $d_{2}$ given by $x_{1}=-a$, where $0<a<1$. Thus, due to symmetry, we have

$$
\begin{aligned}
p_{1} & =\frac{\int_{b}^{\pi-b}(\cos \theta, \sin \theta) \mathrm{d} \theta}{\int_{b}^{\pi-b} \mathrm{~d} \theta}=\left(0, \frac{2 \cos b}{\pi-2 b}\right), \\
p_{2} & =\frac{\frac{1}{4} \int_{-1}^{-a}(x, 0) \mathrm{d} x+\frac{1}{4 \pi} \int_{\pi-b}^{\frac{3 \pi}{2}}(\cos \theta, \sin \theta) \mathrm{d} \theta}{\frac{1}{4} \int_{-1}^{-a} \mathrm{~d} x+\frac{1}{4 \pi} \int_{\pi-b}^{\frac{3 \pi}{2}} \mathrm{~d} \theta} \\
& =\left(\frac{-\pi a^{2}+2 \sin b+\pi+2}{\pi(2 a-3)-2 b},-\frac{2 \cos b}{-2 \pi a+2 b+3 \pi}\right), \\
p_{3} & =(0,0), \quad d_{1}=(-\cos b, \sin b), \text { and } d_{2}=(-a, 0) .
\end{aligned}
$$

Thus, solving the canonical equations $\rho\left(d_{1}, p_{1}\right)-\rho\left(d_{1}, p_{2}\right)=0$, and $\rho\left(d_{2}, p_{2}\right)-$ $\rho\left(d_{2}, p_{3}\right)=0$, we have $a=0.377997, b=0.678642$. Hence, putting the values of $a$ and $b$ we have, $p_{1}=(0,0.872524), p_{2}=(-0.707525,-0.185184)$, and $p_{3}=(0,0)$, and so, due to symmetry, $p_{4}=(0.707525,-0.185184)$. The corresponding distortion error is given by

$$
\begin{aligned}
& V(P ; \alpha)=\frac{1}{4 \pi} \int_{b}^{\pi-b} \rho\left((\cos \theta, \sin \theta), p_{2}\right) \mathrm{d} \theta+ \\
& 2\left(\frac{1}{4} \int_{-1}^{-a} \rho\left((x, 0), p_{2}\right) \mathrm{d} x+\frac{1}{4 \pi} \int_{\pi-b}^{\frac{3 \pi}{2}} \rho\left((\cos \theta, \sin \theta), p_{2}\right) \mathrm{d} \theta\right)+ \\
& \frac{1}{4} \int_{-a}^{a} \rho\left((x, 0), p_{3}\right) \mathrm{d} x=0.21596 .
\end{aligned}
$$

Case 2. $\alpha$ does not contain any point from $L_{1}$, the Voronoi region of which does not contain any point from $L_{2}$.

In this case, we can assume that $p_{1}, p_{2}, p_{3}, p_{4}$ can be located as shown in Figure 2 (ii). Let the boundary of the Voronoi regions of $p_{1}$ and $p_{2}$ intersect $L_{2}$ at the point $d_{1}$ given by the parametric value $\theta=\pi-b$, where $0<b<\frac{\pi}{2}$. Thus, due to symmetry, we have

$$
\begin{aligned}
& p_{1}=\frac{\int_{b}^{\pi-b}(\cos \theta, \sin \theta) \mathrm{d} \theta}{\int_{b}^{\pi-b} \mathrm{~d} \theta}=\left(0, \frac{2 \cos b}{\pi-2 b}\right), \\
& p_{2}=\frac{\frac{1}{4} \int_{-1}^{0}(x, 0) \mathrm{d} x+\frac{1}{4 \pi} \int_{\pi-b}^{\pi+b}(\cos \theta, \sin \theta) \mathrm{d} \theta}{\frac{1}{4} \int_{-1}^{0} \mathrm{~d} x+\frac{1}{4 \pi} \int_{\pi-b}^{\pi+b} \mathrm{~d} \theta}=\left(-\frac{4 \sin b+\pi}{4 b+2 \pi}, 0\right),
\end{aligned}
$$

and

$$
d_{1}=(-\cos b, \sin b)
$$




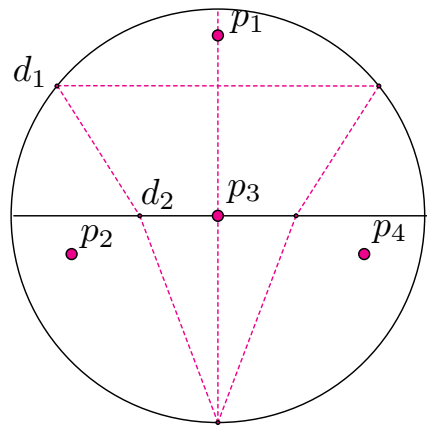

(i)

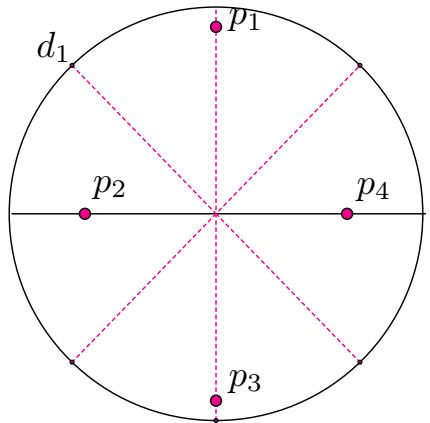

(ii)

FiguRe 2.

Thus, solving the canonical equations $\rho\left(d_{1}, p_{1}\right)-\rho\left(d_{1}, p_{2}\right)=0$, we have $b=$ 0.800791 . Hence, putting the values of $b$, we have, $p_{1}=(0,0.90407), p_{2}=$ $(-0.633881,0)$, and so, due to symmetry, we have $p_{3}=(0.633881,0)$, and $p_{4}=(0,-0.90407)$. The corresponding distortion error is given by

$$
\begin{gathered}
V(P ; \alpha)=2\left(\frac{1}{4 \pi} \int_{b}^{\pi-b} \rho\left((\cos \theta, \sin \theta), p_{2}\right) \mathrm{d} \theta+\frac{1}{4} \int_{-1}^{0} \rho\left((x, 0), p_{2}\right) \mathrm{d} x\right. \\
\left.+\frac{1}{4 \pi} \int_{\pi-b}^{\pi+b} \rho\left((\cos \theta, \sin \theta), p_{2}\right) \mathrm{d} \theta\right)=0.163013
\end{gathered}
$$

Comparing Case 1 and Case 2, we see that if $\alpha$ contains only one point from $L_{1}$, the Voronoi regions of which does not contain any point from $L_{2}$, then the distortion error is larger than the distortion error obtained in Case 2. Similarly, we can show that if $\alpha$ contains more than one point from $L_{1}$, the Voronoi regions of which do not contain any point from $L_{2}$, then the distortion error is larger than the distortion error obtained in Case 2. Considering all the above cases, we see that the distortion error in Case 2 is the smallest. Hence, the points in $\alpha$ obtained in Case 2 form an optimal set of four-means, and the corresponding quantization error is given by $V_{4}=0.163013$. Thus, the proof of the proposition is complete.

Proposition 2.6. An optimal set of five-means is given by

$$
\{(0,0.903584),(-0.788308,0),(0,0),(0,-0.903584),(0.788308,0)\}
$$

and the corresponding quantization error is $V_{5}=0.119779$. 
P r o o f. Let $\alpha:=\left\{p_{1}, p_{2}, p_{3}, p_{4}, p_{5}\right\}$ be an optimal set of five-means. The following cases can arise:

Case 1. $\alpha$ contains two points from $L_{1}$, the Voronoi regions of which do not contain any point from $L_{2}$.

In this case, we can assume that $p_{1}, p_{2}, \ldots, p_{5}$ can be located as shown in Figure 3 (i). Let the boundary of the Voronoi regions of $p_{1}$ and $p_{2}$ intersect $L_{2}$ at the point $d_{1}$ given by the parametric value $\theta=\pi-b$, where $0<b<\frac{\pi}{2}$, and the boundary of the Voronoi regions of $p_{2}$ and $p_{3}$ intersect $L_{1}$ at the point $d_{2}$ given by $x_{1}=-a$, where $0<a<1$. Thus, due to symmetry, we have

$$
\begin{aligned}
p_{1} & =\frac{\int_{b}^{\pi-b}(\cos \theta, \sin \theta) \mathrm{d} \theta}{\int_{b}^{\pi-b} \mathrm{~d} \theta}=\left(0, \frac{2 \cos b}{\pi-2 b}\right), \\
p_{2} & =\frac{\frac{1}{4} \int_{-1}^{-a}(x, 0) \mathrm{d} x+\frac{1}{4 \pi} \int_{\pi-b}^{\frac{3 \pi}{2}}(\cos \theta, \sin \theta) \mathrm{d} \theta}{\frac{1}{4} \int_{-1}^{-a} \mathrm{~d} x+\frac{1}{4 \pi} \int_{\pi-b}^{\frac{3 \pi}{2}} \mathrm{~d} \theta} \\
& =\left(\frac{-\pi a^{2}+2 \sin b+\pi+2}{\pi(2 a-3)-2 b},-\frac{2 \cos b}{-2 \pi a+2 b+3 \pi}\right), \\
p_{3} & =\left(-\frac{a}{2}, 0\right), \quad d_{1}=(-\cos b, \sin b), \quad \text { and } \quad d_{2}=(-a, 0) .
\end{aligned}
$$

Thus, solving the canonical equations $\rho\left(d_{1}, p_{1}\right)-\rho\left(d_{1}, p_{2}\right)=0$, and $\rho\left(d_{2}, p_{2}\right)-$ $\rho\left(d_{2}, p_{3}\right)=0$, we have $a=0.567815, b=0.656426$. Hence, putting the values of $a$ and $b$ we have, $p_{1}=(0,0.866365), p_{2}=(-0.74607,-0.220972)$, and $p_{3}=(-0.283907,0)$, and so, due to symmetry, $p_{4}=(0.283907,0)$, and $p_{5}=$ $(0.74607,-0.220972)$. The corresponding distortion error is given by

$$
\begin{aligned}
V(P ; \alpha)= & \frac{1}{4 \pi} \int_{b}^{\pi-b} \rho\left((\cos \theta, \sin \theta), p_{2}\right) \mathrm{d} \theta+ \\
& 2\left(\frac{1}{4} \int_{-1}^{-a} \rho\left((x, 0), p_{2}\right) \mathrm{d} x+\frac{1}{4 \pi} \int_{\pi-b}^{\frac{3 \pi}{2}} \rho\left((\cos \theta, \sin \theta), p_{2}\right) \mathrm{d} \theta+\right. \\
& \left.\frac{1}{4} \int_{-a}^{0} \rho\left((x, 0), p_{3}\right) \mathrm{d} x\right)=0.18911 .
\end{aligned}
$$

Case 2. $\alpha$ contains only one point from $L_{1}$, the Voronoi region of which does not contain any point from $L_{2}$.

In this case, we can assume that $p_{1}, p_{2}, \ldots, p_{5}$ can be located as shown in Figure 3 (ii). Let the boundary of the Voronoi regions of $p_{1}$ and $p_{2}$ intersect $L_{2}$ at the point $d_{1}$ given by the parametric value $\theta=\pi-b$, where $0<b<\frac{\pi}{2}$, the boundary of the Vonoroi regions of $p_{2}$ and $p_{3}$ intersect $L_{1}$ at the point $d_{2}$ 
given by $x_{1}=-a$, where $0<a<1$. Thus, due to symmetry, we have

$$
\begin{aligned}
& p_{1}=\frac{\int_{b}^{\pi-b}(\cos \theta, \sin \theta) \mathrm{d} \theta}{\int_{b}^{\pi-b} \mathrm{~d} \theta}=\left(0, \frac{2 \cos b}{\pi-2 b}\right), \\
& p_{2}=\frac{\frac{1}{4} \int_{-1}^{-a}(x, 0) \mathrm{d} x+\frac{1}{4 \pi} \int_{\pi-b}^{\pi+b}(\cos \theta, \sin \theta) \mathrm{d} \theta}{\frac{1}{4} \int_{-1}^{-a} \mathrm{~d} x+\frac{1}{4 \pi} \int_{\pi-b}^{\pi+b} \mathrm{~d} \theta}=\left(-\frac{-\pi a^{2}+4 \sin b+\pi}{-2 \pi a+4 b+2 \pi}, 0\right), \\
& p_{3}=(0,0), \quad d_{1}=(-\cos b, \sin b), \quad d_{2}=(-a, 0) .
\end{aligned}
$$

Thus, solving the canonical equations $\rho\left(d_{1}, p_{1}\right)-\rho\left(d_{1}, p_{2}\right)=0, \rho\left(d_{2}, p_{2}\right)-$ $\rho\left(d_{2}, p_{3}\right)=0$, we have $a=0.394154$, and $b=0.798783$. Hence, putting the values of $a$, and $b$, we have, $p_{1}=(0,0.903584), p_{2}=(-0.788308,0)$, and $p_{3}=(0,0)$, and so, due to symmetry, $p_{4}=(0,-0.903584)$, and $p_{5}=(0.788308,0)$. The corresponding distortion error is given by

$$
\begin{aligned}
& V(P ; \alpha)=2( \frac{1}{4 \pi} \int_{b}^{\pi-b} \rho\left((\cos \theta, \sin \theta), p_{2}\right) \mathrm{d} \theta+ \\
&\left.\frac{1}{4} \int_{-1}^{-a} \rho\left((x, 0), p_{2}\right) \mathrm{d} x+\frac{1}{4 \pi} \int_{\pi-b}^{\pi+b} \rho\left((\cos \theta, \sin \theta), p_{2}\right) \mathrm{d} \theta\right)+ \\
& \frac{1}{4} \int_{-a}^{a} \rho\left((x, 0), p_{3}\right) \mathrm{d} x=0.119779 .
\end{aligned}
$$

Case 3. $\alpha$ does not contain any point from $L_{1}$, the Voronoi region of which does not contain any point from $L_{2}$.

In this case, we can assume that $p_{1}, p_{2}, \ldots, p_{5}$ can be located as shown in Figure 3 (iii). Let the boundary of the Voronoi regions of $p_{1}$ and $p_{2}$ intersect $L_{2}$ at the point $d_{1}$ given by the parametric value $\theta=\pi-b$, where $0<b<\frac{\pi}{2}$, and the boundary of the Voronoi regions of $p_{2}$ and $p_{3}$ intersect $L_{2}$ as the point $d_{2}$ given by the parametric value $\theta=\pi+c$, where $0<c<\frac{\pi}{2}$. Thus, due to symmetry, we have

$$
\begin{aligned}
p_{1} & =\frac{\int_{\frac{\pi}{2}}^{\pi-b}(\cos \theta, \sin \theta) \mathrm{d} \theta}{\int_{\frac{\pi}{2}}^{\pi-b} \mathrm{~d} \theta}=\left(\frac{2(\sin b-1)}{\pi-2 b}, \frac{2 \cos b}{\pi-2 b}\right), \\
p_{2} & =\frac{\frac{1}{4} \int_{-1}^{0}(x, 0) \mathrm{d} x+\frac{1}{4 \pi} \int_{\pi-b}^{\pi+c}(\cos \theta, \sin \theta) \mathrm{d} \theta}{\frac{1}{4} \int_{-1}^{0} \mathrm{~d} x+\frac{1}{4 \pi} \int_{\pi-b}^{\pi+c} \mathrm{~d} \theta} \\
& =\left(-\frac{2 \sin b+2 \sin c+\pi}{2(b+c+\pi)}, \frac{\cos c-\cos b}{b+c+\pi}\right),
\end{aligned}
$$




$$
\begin{aligned}
& p_{3}=\frac{\int_{\pi+c}^{2 \pi-c}(\cos \theta, \sin \theta) \mathrm{d} \theta}{\int_{\pi+c}^{2 \pi-c} \mathrm{~d} \theta}=\left(0,-\frac{2 \cos c}{\pi-2 c}\right), \\
& d_{1}=(-\cos b, \sin b), \text { and } d_{2}=(-\cos c,-\sin c) .
\end{aligned}
$$

Thus, solving the canonical equations $\rho\left(d_{1}, p_{1}\right)-\rho\left(d_{1}, p_{2}\right)=0$, and $\rho\left(d_{2}, p_{2}\right)-$ $\rho\left(d_{2}, p_{3}\right)=0$, we have $b=0.426473$, and $c=0.837847$. Hence, putting the values of $b$, and $c$, we have, $p_{1}=(-0.512388,0.795606), p_{2}=(-0.619091,-0.0547824)$, $p_{3}=(0,-0.912839)$, and so, due to symmetry, $p_{4}=(0.619091,-0.0547824)$, and $p_{5}=(0.512388,0.795606)$. The corresponding distortion error is given by

$$
\begin{aligned}
& V(P ; \alpha) \\
& =2\left(\frac{1}{4 \pi} \int_{\frac{\pi}{2}}^{\pi-b} \rho\left((\cos \theta, \sin \theta), p_{2}\right) \mathrm{d} \theta\right)+\frac{1}{4} \int_{-1}^{0} \rho\left((x, 0), p_{2}\right) \mathrm{d} x \\
& \left.\quad+\frac{1}{4 \pi} \int_{\pi-b}^{\pi+c} \rho\left((\cos \theta, \sin \theta), p_{2}\right) \mathrm{d} \theta\right)+\frac{1}{4 \pi} \int_{\pi+c}^{2 \pi-c} \rho\left((\cos \theta, \sin \theta), p_{3}\right) \mathrm{d} \theta \\
& =0.1355 .
\end{aligned}
$$

Comparing Case 1 and Case 2, we see that if $\alpha$ contains two points from $L_{1}$, the Voronoi regions of which do not contain any point from $L_{2}$, then the distortion error is larger than the distortion error obtained in Case 2. Similarly, we can show that if $\alpha$ contains more than two points from $L_{1}$, the Voronoi regions of which do not contain any point from $L_{2}$, then the distortion error is larger than the distortion error obtained in Case 2. Comparing Case 2 and Case 3, we see that Case 3 can not happen as the distortion error is larger in Case 3. Considering all the above cases, we see that the distortion error in Case 2 is the smallest. Hence, the points in $\alpha$ obtained in Case 2 form an optimal set of five-means, and the corresponding quantization error is given by $V_{5}=0.119779$. Thus, the proof of the proposition is complete.

Proposition 2.7. An optimal set of six-means is

$$
\begin{array}{r}
\{(-0.497577,0.809422),(-0.786245,-0.0706781),(0,0),(0,-0.913921), \\
(0.786245,-0.0706781),(0.497577,0.809422)\}
\end{array}
$$

and the corresponding quantization error for six-means is given by $V_{6}=0.093342$.

Proof. Let $\alpha:=\left\{p_{1}, p_{2}, p_{3}, p_{4}, p_{5}, p_{6}\right\}$ be an optimal set of six-means. As in Proposition 2.6. here also we consider three different cases as shown in Figure 4. 


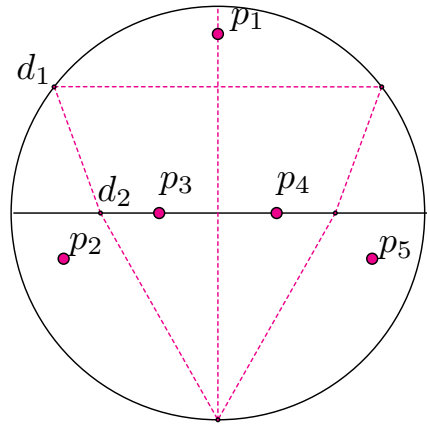

(i)

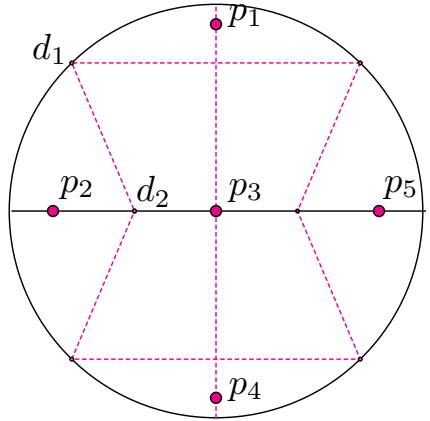

(ii)

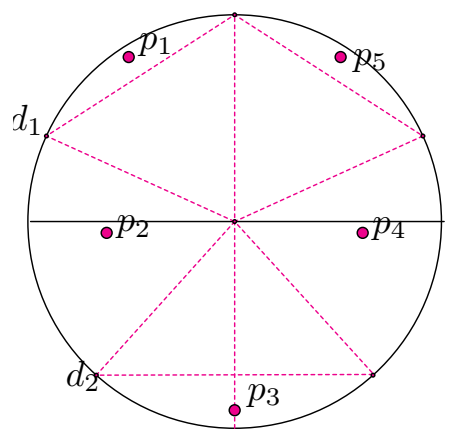

(iii)

FiguRE 3.

In each case, we calculate the distortion errors. Then, comparing the distortion errors, we see that the points given by the proposition give the smallest distortion error for six points, and hence they form an optimal set of six-means, which is shown by Figure 4 (ii). Thus, the proof of the proposition is deduced.

Proceeding in the similar way as Proposition 2.6 and Proposition 2.7 we can deduce that the following proposition is also true.

Proposition 2.8. Let $\alpha_{n}$ be an optimal set of $n$-means, and let $V_{n}$ be the corresponding quantization error. Then,

$$
\begin{array}{r}
\alpha_{7}=\{(-0.476891,0.827476),(-0.788772,0),(0,0),(-0.476891,-0.827476), \\
(0.476891,-0.827476),(0.788772,0),(0.476891,0.827476)\} \\
\text { with } V_{7}=0.070674, \text { see Figure } 5(\mathrm{i}) ;
\end{array}
$$




\section{MRINAL KANTI ROYCHOWDHURY — WASIELA SALINAS}

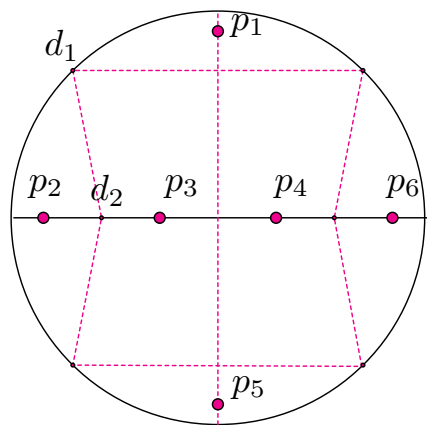

(i)

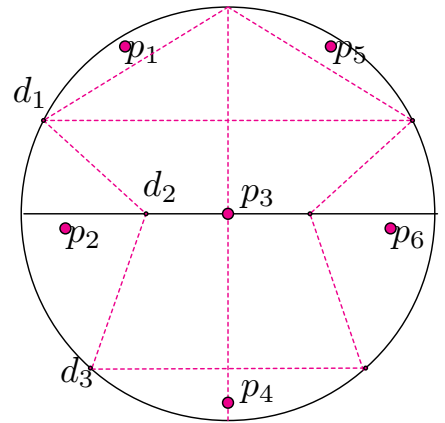

(ii)

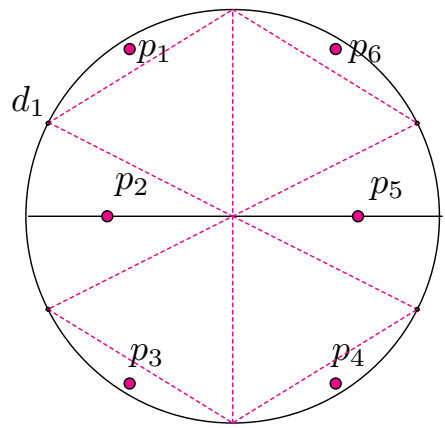

(iii)

FiguRe 4.

$$
\begin{aligned}
\alpha_{8}=\{ & (-0.475258,0.828843),(-0.860649,0), \\
& (-0.286883,0),(-0.475258,-0.828843),(0.475258,-0.828843), \\
& (0.860649,0),(0.286883,0),(0.475258,0.828843)\}
\end{aligned}
$$$$
\text { with } V_{8}=0.0577852 \text {, see Figure } 5 \text { (ii); }
$$

$$
\begin{gathered}
\alpha_{9}=\{-0.463928,0.838108),(-0.857223,0.0396484),(-0.286659,0), \\
(-0.704114,-0.671446),(0,-0.972943),(0.704114,-0.671446), \\
(0.286659,0),(0.857223,0.0396484),(0.463928,0.838108)\}
\end{gathered}
$$$$
\text { with } V_{9}=0.04803 \text {, see Figure } 5 \text { (iii); }
$$

$$
\begin{aligned}
\alpha_{10}= & \{(0,0.974386),(-0.690161,0.687826),(-0.854308,0),(-0.284769,0), \\
& (-0.690161,-0.687826),(0,-0.974386),(0.690161,-0.687826),
\end{aligned}
$$

$(0.854308,0),(0.284769,0),(0.690161,0.687826)\}$,

with $V_{10}=0.039046$, see Figure 5 (iv). 


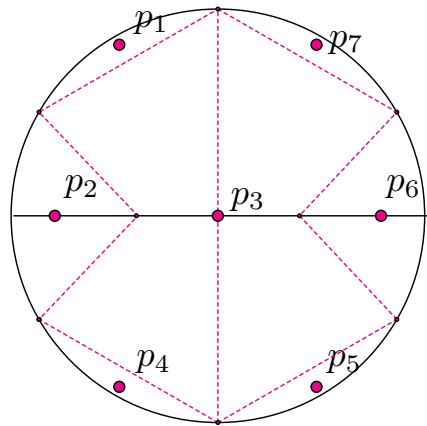

(i)

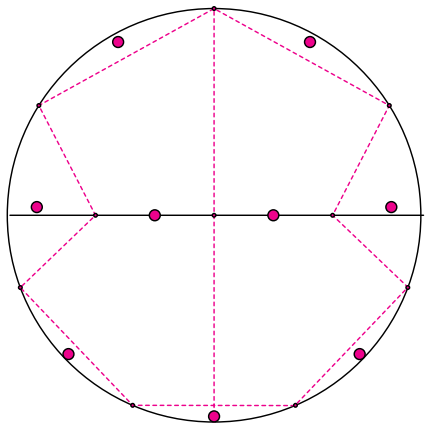

(iii)

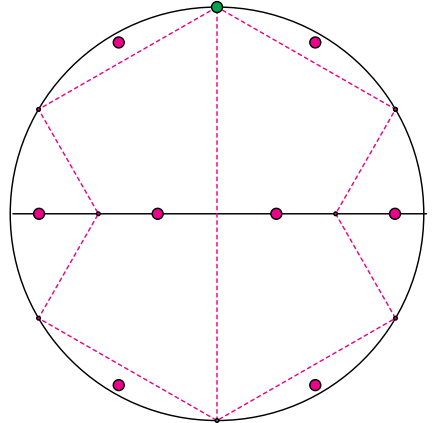

(ii)

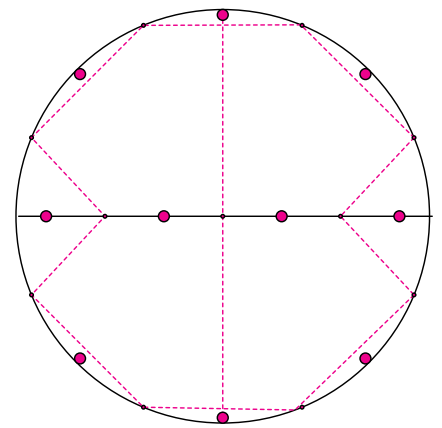

(iv)

FiguRE 5 .

The following proposition plays an important role in the paper.

Proposition 2.9. Let $\alpha_{n}$ be an optimal set of $n$-means for $P$, and $n \geq 5$. Then, $\alpha_{n}$ contains at least one point from $L_{1}$, the Voronoi region of which does not contain any point from $L_{2}$; and at least one point from $L_{2}$, the Voronoi region of which does not contain any point from $L_{1}$.

Proof. Let $V_{n}$ denote the $n$th quantization error for any positive integer $n$. By the previous propositions, the lemma is true for $5 \leq n \leq 10$. Let $n \geq 11$. Then, $V_{n} \leq V_{11}<V_{10}=0.039046$. For the sake of contradiction, assume that for $n \geq 11$, the set $\alpha_{n}$ does not contain any point from $L_{1}$, the Voronoi region of which does not contain any point from $L_{2}$. Then,

$$
\begin{aligned}
V_{n} & >\int_{L_{1}} \min _{a \in\left\{\left(-\frac{1}{2}, 0\right),\left(0, \frac{1}{2}\right)\right\}} \rho((x, 0), a) \mathrm{d} P \\
& =\frac{1}{4} \int_{-1}^{0} \rho\left((t, 0),\left(-\frac{1}{2}, 0\right)\right) \mathrm{d} t+\frac{1}{4} \int_{0}^{1} \rho\left((t, 0),\left(\frac{1}{2}, 0\right)\right) \mathrm{d} t=\frac{1}{24},
\end{aligned}
$$


implying $V_{n}>\frac{1}{24}=0.0416667>V_{10}$, which leads to a contradiction. Hence, $\alpha_{n}$ contains at least one point from $L_{1}$, the Voronoi region of which does not contain any point from $L_{2}$. Similarly, we can prove the other part of the proposition. Thus, the proof of the proposition is complete.

We now state and prove the following theorem, which is the main theorem of this section. Notice that we are saying the theorem as the main theorem of this section, because as mentioned in Remark 2.11, this theorem helps us to calculate all the optimal sets of $n$-means, and so, the $n$th quantization errors for all $n \geq 5$ for the mixed distribution $P$.

Theorem 2.10. Let $n \geq 5$ be a positive integer, and let $\alpha_{n}$ be an optimal set of $n$-means for $P$. Let $3 k+2 \leq n \leq 3 k+4$ for some positive integer $k$. Then, $\alpha_{n}$ contains $k$ elements from $L_{1}$, the Voronoi regions of which do not contain any point from $L_{2}$.

Proof. By Proposition 2.9, for $n \geq 5$, the set $\alpha_{n}$ always contains points from $L_{1}$, the Voronoi regions of which do not contain any point from $L_{2}$, and points from $L_{2}$, the Voronoi regions of which do not contain any point from $L_{1}$. Since the Voronoi region of a point in an optimal set covers maximum area within a shortest distance $P$-almost surely, the set $\alpha_{n}$, given in the theorem, must contain the two points, the Voronoi regions of which contain points from both $L_{1}$ and $L_{2}$, in other words, the Voronoi regions of these two points contain points around the two intersections of $L_{1}$ and $L_{2}$. Each of the remaining $n-2$ points occurs due to the uniform distribution on $L_{1}$, or $L_{2}$, the Voronoi region of which contains points only from $L_{1}$, or from $L_{2}$, respectively.

Let $n=n_{1}+n_{2}+k+2$ be such that $\alpha_{n}$ contains $k$ elements from $L_{1}$, the Voronoi regions of which do not contain any point from $L_{2} ; n_{1}$ elements from above the $x_{1}$-axis, the Voronoi regions of which do not contain any point from $L_{1}$, and $n_{2}$ elements from below the $x_{1}$-axis, the Voronoi regions of which do not contain any point from $L_{1}$. Then, there exist three real numbers $a, b$, and $c$, where $-1<a<1,0<b<\frac{\pi}{2}$, and $0<c<\frac{\pi}{2}$, such that the following occur:

(i) The $k$ elements that $\alpha_{n}$ contains from $L_{1}$ occur due to the uniform distribution on $[-a, a]$, and as mentioned in Theorem 1.2 , are given by the set

$$
\left\{-a+\frac{2 i-1}{k} a: 1 \leq i \leq k\right\}
$$

with distortion error given by

$k\left(\right.$ distortion error due to the point $-a+\frac{a}{k}$ in the interval $\left.\left[-a,-a+\frac{2 a}{k}\right]\right)$

$$
=\frac{k}{4} \int_{-a}^{-a+\frac{2 a}{k}}\left(t-\left(-a+\frac{a}{k}\right)\right)^{2} \mathrm{~d} t=\frac{a^{3}}{6 k^{2}} .
$$




\section{QUANTIZATION FOR A MIXTURE OF UNIFORM DISTRIBUTIONS}

(ii) The $n_{1}$ elements that $\alpha_{n}$ contains from above the $x_{1}$-axis, the Voronoi regions of which do not contain any point from $L_{1}$, occur due to the uniform distribution on the circular $\operatorname{arc}\{(\cos \theta, \sin \theta): b \leq \theta \leq \pi-b\}$, and by Theorem 1.3, are given by the set

$$
\begin{aligned}
& \left\{\frac{2 n_{1}}{\pi-2 b} \sin \frac{\pi-2 b}{2 n_{1}}\left(\cos \left(b+(2 j-1) \frac{\pi-2 b}{2 n_{1}}\right), \sin \left(b+(2 j-1) \frac{\pi-2 b}{2 n_{1}}\right)\right)\right. \\
& \left.: 1 \leq j \leq n_{1}\right\}
\end{aligned}
$$

with distortion error

$$
\begin{aligned}
n_{1}\left(\frac{1}{4 \pi} \int_{b}^{b+\frac{\pi-2 b}{n_{1}} \rho(}(\cos \theta, \sin \theta), \frac{2 n_{1}}{\pi-2 b} \sin \left(\frac{\pi-2 b}{2 n_{1}}\right)\right. \\
\left.\left.\left(\cos \left(b+\frac{\pi-2 b}{2 n_{1}}\right), \sin \left(b+\frac{\pi-2 b}{2 n_{1}}\right)\right)\right) \mathrm{d} \theta\right) \\
=\frac{(\pi-2 b)^{2}-2 n_{1}^{2}+2 n_{1}^{2} \cos \left(\frac{2 b-\pi}{n_{1}}\right)}{4 \pi(\pi-2 b)},
\end{aligned}
$$

and we denote it by $D_{n_{1}}$.

(iii) The $n_{2}$ elements that $\alpha_{n}$ contains from below the $x_{1}$-axis, the Voronoi regions of which do not contain any point from $L_{1}$, occur due to the uniform distribution on the circular arc $\{(\cos \theta, \sin \theta): \pi+c \leq \theta \leq 2 \pi-c\}$, and by Theorem 1.3. are given by the set

$$
\begin{aligned}
& \qquad\left\{\frac{2 n_{2}}{\pi-2 c} \sin \frac{\pi-2 c}{2 n_{2}}\left(\cos \left(\pi+c+(2 j-1) \frac{\pi-2 c}{2 n_{2}}\right), \sin \left(\pi+c+(2 j-1) \frac{\pi-2 c}{2 n_{2}}\right)\right)\right. \\
& \text { with distortion error }
\end{aligned}
$$

$$
\begin{aligned}
n_{2}\left(\frac{1}{4 \pi} \int_{\pi+c}^{\pi+c+\frac{\pi-2 c}{n_{2}} \rho}(\right. & (\cos \theta, \sin \theta), \frac{2 n_{2}}{\pi-2 c} \sin \left(\frac{\pi-2 c}{2 n_{2}}\right) \\
& \left.\left.\left(\cos \left(\pi+c+\frac{\pi-2 c}{2 n_{2}}\right), \sin \left(\pi+c+\frac{\pi-2 c}{2 n_{2}}\right)\right)\right) \mathrm{d} \theta\right) \\
= & \frac{(\pi-2 c)^{2}-2 n_{2}^{2}+2 n_{2}^{2} \cos \left(\frac{2 c-\pi}{n_{2}}\right)}{4 \pi(\pi-2 c)}
\end{aligned}
$$

and we denote it by $D_{n_{2}}$. 


\section{MRINAL KANTI ROYCHOWDHURY — WASIELA SALINAS}

(iv) The two points in $\alpha_{n}$, the Voronoi regions of which contain points from both $L_{1}$ and $L_{2}$, are given by the set $\{(-r, s),(r, s)\}$, where

$$
\begin{aligned}
(-r, s) & =\frac{\frac{1}{4} \int_{-1}^{-a}(t, 0) \mathrm{d} t+\frac{1}{4 \pi} \int_{\pi-b}^{\pi+c}(\cos \theta, \sin \theta) \mathrm{d} \theta}{\frac{1}{4} \int_{-1}^{-a} \mathrm{~d} t+\frac{1}{4 \pi} \int_{\pi-b}^{\pi+c} \mathrm{~d} \theta} \\
& =\left(-\frac{-\pi a^{2}+2 \sin b+2 \sin c+\pi}{2(-\pi a+b+c+\pi)}, \frac{\cos c-\cos b}{-\pi a+b+c+\pi}\right),
\end{aligned}
$$

i.e.,

$$
r=\frac{-\pi a^{2}+2 \sin b+2 \sin c+\pi}{2(-\pi a+b+c+\pi)}, \quad \text { and } \quad s=\frac{\cos c-\cos b}{-\pi a+b+c+\pi},
$$

and the distortion error for both the two points is given by

$$
\begin{aligned}
2( & \left.\frac{1}{4} \int_{-1}^{-a} \rho((t, 0),(-r, s)) \mathrm{d} t+\frac{1}{4 \pi} \int_{\pi-b}^{\pi+c} \rho((\cos \theta, \sin \theta),(-r, s)) \mathrm{d} \theta\right) \\
= & \frac{1}{24 \pi(-\pi a+b+c+\pi)}\left(\pi^{2} a^{4}-4 \pi a^{3} b-4 \pi a^{3} c-4 \pi^{2} a^{3}\right. \\
& +12 \pi\left(a^{2}-1\right) \sin b+12 \pi a^{2} \sin c+6 \pi^{2} a^{2} \\
& -12 \pi a b-12 \pi a c-4 \pi^{2} a+12 b^{2}+24 b c \\
& \left.+24 \cos (b+c)+16 \pi b+12 c^{2}+16 \pi c-12 \pi \sin c+\pi^{2}-24\right),
\end{aligned}
$$

and we denote it by $D(a, b, c)$.

Let $V\left(n_{1}, n_{2}, k\right)$ denote the distortion error due to the all above $n_{1}+n_{2}+k+2$ elements in $\alpha_{n}$. Then, we have

$$
V\left(n_{1}, n_{2}, k\right)=\frac{a^{3}}{6 k^{2}}+D_{n_{1}}+D_{n_{2}}+D(a, b, c) .
$$

Let $n_{1}, n_{2}$, and $k$ be fixed. Then, using the partial derivatives we can obtain the following equations

$$
\frac{\partial}{\partial a}\left(V\left(n_{1}, n_{2}, k\right)\right)=0, \quad \frac{\partial}{\partial b}\left(V\left(n_{1}, n_{2}, k\right)\right)=0, \quad \text { and } \quad \frac{\partial}{\partial c}\left(V\left(n_{1}, n_{2}, k\right)\right)=0 .
$$

For a given set of values of $n_{1}, n_{2}$, and $k$, solving the equations in (2), we can obtain the values of $a, b, c$. Putting the values of $a, b, c$ in (11), we can obtain the distortion error for the given set of values of $n_{1}, n_{2}, k$.

Now, to prove the theorem we use induction on $k$. If $k=1$, and $k=2$, the theorem is true due to the previous propositions. Let us assume that the theorem is true for $k=m$, i.e., when $3 m+2 \leq n \leq 3 m+4$. We now prove that the theorem is true for $3(m+1)+2 \leq n \leq 3(m+1)+4$. By the assumption, the theorem is true for $n=3 m+4$, i.e., the set $\alpha_{3 m+4}$ contains 


\section{QUANTIZATION FOR A MIXTURE OF UNIFORM DISTRIBUTIONS}

$m$ points from $L_{1}$, the Voronoi regions of which do not contain any point from $L_{2}$, and $(2 m+2)$ points occur due to the uniform distribution on $L_{2}$, the Voronoi region of which do not contain any point from $L_{1}$. Again, due to the mixed distribution with equal weights to the component probabilities, and symmetry of the circle with respect to the $x_{1}$-axis, we can can assume that $\alpha_{n}$ contains $m+1$ elements from above, and $m+1$ elements from below. Now, to calculate $\alpha_{n+1}$, we need to add one extra point either to $L_{1}$, or $L_{2}$ in an optimal way, i.e., the Voronoi regions of the new point will contain only the points from $L_{1}$, or from $L_{2}$, and the overall distortion error due to $n+1$ points becomes smallest. First suppose that the extra point is added to $L_{1}$, the Voronoi region of which does not contain any point from $L_{2}$. As described above using (10), we calculate the distortion error $V(m+1, m+1, m+1)$. Next, suppose that the extra point is added to $L_{2}$, the Voronoi region of which does not contain any point from $L_{1}$, and using (11), we calculate the distortion error $V(m+2, m+1, m)$, or $V(m+1, m+2, m)$. We see that the distortion error $V(m+1, m+1, m+1)$ is the smallest, which implies the fact that $\alpha_{n+1}$ contains $m+1$ points from $L_{1}$. Once, $\alpha_{n+1}$ is known, similarly we can obtain $\alpha_{n+2}$, and $\alpha_{n+3}$ with distortion errors, respectively, $V(m+1, m, m+1)$ and $V(m+1, m+1, m+1)$. Thus, we see that each of $\alpha_{n+1}, \alpha_{n+2}$, and $\alpha_{n+3}$ contains $m+1$ points from $L_{1}$, the Voronoi regions of which do not contain any point from $L_{2}$. Notice that $n+1=3(m+1)+2, n+2=3(m+1)+3$, and $n+1=3(m+1)+4$, i.e., for the positive integer $n$ satisfying $3(m+1)+2 \leq n \leq 3(m+1)+4$, the set $\alpha_{n}$ contains $m+1$ elements from $L_{1}$, the Voronoi regions of which do not contain any point from $L_{2}$. Thus, the theorem is true for $k=m+1$ if it is true for $k=m$. Hence, by the principle of mathematical induction, the theorem is true for all positive integers $k$, and thus, the proof of the theorem is complete.

REMARK 2.11. For $n \geq 5$, let $3 k+2 \leq n \leq 3 k+4$ for some positive integer $k$. Then, by Theorem 2.10, we can say that if $n-k-2$ is an even number, then an optimal set of $n$-means contains $\frac{1}{2}(n-k-2)$ elements from either side of the $x_{1}$-axis, the Voronoi regions of which do not contain any point from $L_{1}$; and if $n-k-2$ is an odd number, then an optimal set of $n$-means contains $\frac{1}{2}\lfloor n-k-2\rfloor$ elements from one side of the $x_{1}$-axis, and $\frac{1}{2}\lfloor n-k-2\rfloor+1$ elements from the other side of the $x_{1}$-axis, the Voronoi regions of which do not contain any point from $L_{1}$. Thus, by Theorem 2.10, using Theorem [1.2, and Theorem 1.3, we can easily determine the optimal sets of $n$-means and the $n$th quantization errors for all $n \geq 5$.

The following proposition gives the quantization dimension and the quantization coefficient for the mixed distribution. 


\section{MRINAL KANTI ROYCHOWDHURY — WASIELA SALINAS}

Proposition 2.12. Quantization dimension $D(P)$ of the mixed distribution $P$ is one, which is the dimension of the underlying space, and the quantization coefficient exists as a finite positive number which equals $\frac{3}{8}\left(4+\pi^{2}\right)$.

Pr o of. By Remark 2.11, we see that if $n$ is of the form $n=3 k+2$ for some positive integer $k$, then $\alpha_{n}$ contains $k$ elements from $L_{1}$, the Voronoi regions of which do not contain any point from $L_{2}$, and $k$ elements from the above, and $k$ elements from below the $x_{1}$-axis, the Voronoi region of which do not contain any point from $L_{1}$. For $n \in \mathbb{N}, n \geq 5$, let $\ell(n)$ be the unique positive integer such that $3 \ell(n)+2 \leq n<3(\ell(n)+1)+2$. Then, $V_{3(\ell(n)+1)+2}<V_{n} \leq V_{3 \ell(n)+2}$ implying

$$
\frac{2 \log (3 \ell(n)+2)}{-\log V_{3(\ell(n)+1)+2}}<\frac{2 \log n}{-\log V_{n}}<\frac{2 \log (3(\ell(n)+1)+2)}{-\log V_{3 \ell(n)+2}} .
$$

Notice that if $n \rightarrow \infty$, then $\ell(n) \rightarrow \infty$. Moreover, if $n \rightarrow \infty$, they by (11) and (2), we can see that $a \rightarrow 1, b \rightarrow 0$, and $c \rightarrow 0$. Assume that $n$ is sufficiently large, in other words, assume that $\ell(n)$ is sufficiently large, and then as $a \rightarrow 1, b \rightarrow 0$, and $c \rightarrow 0$, by (1) we have $D(a, b, c) \rightarrow 0$, implying

$$
V_{3 \ell(n)+2}=V(\ell(n), \ell(n), \ell(n))=\frac{-6 \ell(n)^{4}+6 \ell(n)^{4} \cos \frac{\pi}{\ell(n)}+3 \pi^{2} \ell(n)^{2}+\pi^{2}}{6 \pi^{2} \ell(n)^{2}},
$$

yielding

$$
\begin{aligned}
& \lim _{n \rightarrow \infty} \frac{2 \log (3 \ell(n)+2)}{-\log V_{3(\ell(n)+1)+3}} \\
& =\lim _{\ell(n) \rightarrow \infty} \frac{2 \log (3 \ell(n)+2)}{-\log \left(\frac{-6(\ell(n)+1)^{4}+3 \pi^{2}(\ell(n)+1)^{2}+6(\ell(n)+1)^{4} \cos \left(\frac{\pi}{\ell(n)+1}\right)+\pi^{2}}{6 \pi^{2}(\ell(n)+1)^{2}}\right)}=1,
\end{aligned}
$$

and

$$
\begin{aligned}
& \lim _{n \rightarrow \infty} \frac{2 \log (3(\ell(n)+1)+2)}{-\log V_{3 \ell(n)+2}} \\
& =\lim _{\ell(n) \rightarrow \infty} \frac{2 \log (3(\ell(n)+1)+2)}{-\log \left(\frac{-6 \ell(n)^{4}+6 \ell(n)^{4} \cos \left(\frac{\pi}{\ell(n)}\right)+3 \pi^{2} \ell(n)^{2}+\pi^{2}}{6 \pi^{2} \ell(n)^{2}}\right)}=1
\end{aligned}
$$

and hence, by (3),

$$
\lim _{n \rightarrow \infty} \frac{2 \log n}{-\log V_{n}}=1
$$

which is the dimension of the underlying space.

Again,

$$
(3 \ell(n)+2)^{2} V_{3(\ell(n)+1)+2}<n^{2} V_{n}<(3(\ell(n)+1)+2)^{2} V_{3 \ell(n)+2} .
$$


We have

$$
\begin{aligned}
& \lim _{n \rightarrow \infty}(3 \ell(n)+2)^{2} V_{3(\ell(n)+1)+2} \\
= & \lim _{\ell(n) \rightarrow \infty}(3 \ell(n)+2)^{2} \\
& \frac{-6(\ell(n)+1)^{4}+3 \pi^{2}(\ell(n)+1)^{2}+6(\ell(n)+1)^{4} \cos \left(\frac{\pi}{\ell(n)+1}\right)+\pi^{2}}{6 \pi^{2}(\ell(n)+1)^{2}} \\
= & \frac{3}{8}\left(4+\pi^{2}\right),
\end{aligned}
$$

and

$$
\begin{aligned}
& \lim _{n \rightarrow \infty}(3(\ell(n)+1)+2)^{2} V_{3 \ell(n)+2} \\
& =\lim _{\ell(n) \rightarrow \infty}(3(\ell(n)+1)+2)^{2-6 \ell(n)^{4}+6 \ell(n)^{4} \cos \left(\frac{\pi}{\ell(n)}\right)+3 \pi^{2} \ell(n)^{2}+\pi^{2}} \\
& =\frac{3}{8}\left(4+\pi^{2} \ell(n)^{2}\right.
\end{aligned}
$$

and hence, by (41) we have

$$
\lim _{n \rightarrow \infty} n^{2} V_{n}=\frac{3}{8}\left(4+\pi^{2}\right),
$$

i.e., the quantization coefficient exists as a finite positive number which equals $=\frac{3}{8}\left(4+\pi^{2}\right)$. Thus, the proof of the proposition is complete.

\section{Optimal quantization for the mixture of two uniform distributions on two disconnected line segments}

Let $P_{1}$ and $P_{2}$ be two uniform distributions, respectively, on the intervals $\left[0, \frac{1}{2}\right]$ and $\left[\frac{3}{4}, 1\right]$. Write

$$
J_{1}:=\left[0, \frac{1}{2}\right], \quad \text { and } \quad J_{2}:=\left[\frac{3}{4}, 1\right] .
$$

Let $f_{1}$ and $f_{2}$ be their respective density functions. Then, $f_{1}(x)=2$ if $x \in$ $\left[0, \frac{1}{2}\right]$, and zero, otherwise; and $f_{2}(x)=4$ if $x \in\left[\frac{3}{4}, 1\right]$, and zero, otherwise. Let $P:=\frac{3}{4} P_{1}+\frac{1}{4} P_{2}$. In the sequel, for the mixed distribution $P$, we determine the optimal sets of $n$-means and the $n$th quantization errors for all positive integers $n$. By $E(P)$ and $V(P)$, we mean the expectation and the variance of a random variable with distribution $P$. By $\alpha_{n}(\mu)$, we denote an optimal set of $n$-means with respect to a probability distribution $\mu$, and $V_{n}(\mu)$ represents the corresponding quantization error for $n$-means. If $\mu$ is the mixed distribution $P$, 


\section{MRINAL KANTI ROYCHOWDHURY — WASIELA SALINAS}

in the sequel, we sometimes denote it by $\alpha_{n}$ instead of $\alpha_{n}(P)$, and the corresponding quantization error is denoted by $V_{n}$ instead of $V_{n}(P)$.

Lemma 3.1. Let $P$ be the mixed distribution defined by $P=\frac{3}{4} P_{1}+\frac{1}{4} P_{2}$. Then,

$$
E(P)=\frac{13}{32}, \quad \text { and } \quad V(P)=\frac{277}{3072} \text {. }
$$

Proof. We have

$E(P)=\int x \mathrm{~d} P=\frac{3}{4} \int x \mathrm{~d}\left(P_{1}(x)\right)+\frac{1}{4} \int x \mathrm{~d}\left(P_{2}(x)\right)=\frac{3}{4} \int_{0}^{\frac{1}{2}} 2 x \mathrm{~d} x+\frac{1}{4} \int_{\frac{3}{4}}^{1} 4 x \mathrm{~d} x$

yielding $E(P)=\frac{13}{32}$, and

$V(P)=\int(x-E(P))^{2} \mathrm{~d} P=\frac{3}{4} \int(x-E(P))^{2} \mathrm{~d}\left(P_{1}(x)\right)+\frac{1}{4} \int(x-E(P))^{2} \mathrm{~d}\left(P_{2}(x)\right)$,

implying $V(P)=\frac{277}{3072}$, and thus, the lemma is yielded.

REMARK 3.2. The optimal set of one-mean is the set $\left\{\frac{13}{32}\right\}$, and the corresponding quantization error is the variance $V:=V(P)$ of a random variable with distribution $P$.

LEMma 3.3. The set $\alpha:=\left\{\frac{1}{4}, \frac{7}{8}\right\}$ is an optimal set of two-means, and the corresponding quantization error is given by $V_{2}=\frac{13}{768}$.

Pr o of. Consider the set of two points $\beta$ given by $\beta:=\left\{\frac{1}{4}, \frac{7}{8}\right\}$. The distortion error due to the set $\beta$ is given by

$$
\begin{aligned}
\int \min _{a \in \beta}(x-a)^{2} \mathrm{~d} P & =\int_{J_{1}}\left(x-\frac{1}{4}\right)^{2} \mathrm{~d} P+\int_{J_{2}}\left(x-\frac{7}{8}\right)^{2} \mathrm{~d} P \\
& =\frac{3}{4} \int_{0}^{\frac{1}{2}} 2\left(x-\frac{1}{4}\right)^{2} \mathrm{~d} x+\frac{1}{4} \int_{\frac{3}{4}}^{1} 4\left(x-\frac{7}{8}\right)^{2} \mathrm{~d} x \\
& =\frac{13}{768}=0.0169271 .
\end{aligned}
$$

Since $V_{2}$ is the quantization error for two-means, we have $V_{2} \leq 0.0169271$. Let $\alpha:=\left\{a_{1}, a_{2}\right\}$ be an optimal set of two-means. Since the points in an optimal set are the conditional expectations in their own Voronoi regions, without any loss of generality, we can assume that $0<a_{1}<a_{2}<1$. We now show that the 


\section{QUANTIZATION FOR A MIXTURE OF UNIFORM DISTRIBUTIONS}

Voronoi region of $a_{1}$ does not contain any point from $J_{2}$, and the Voronoi region of $a_{2}$ does not contain any point from $J_{1}$. Suppose that $\frac{13}{40} \leq a_{1}$. Then,

$$
V_{2}>\int_{\left[0, \frac{13}{40}\right]}\left(x-\frac{13}{40}\right)^{2} \mathrm{~d} P=\frac{2197}{128000}=0.0171641>V_{2},
$$

which is a contradiction, and so, we can assume that $a_{1}<\frac{13}{40}<\frac{1}{2}$.

Since $a_{1}<\frac{13}{40}$, the Voronoi region of $a_{1}$ does not contain any points from $J_{2}$. If it contains points from $J_{2}$, then $\frac{1}{2}\left(a_{1}+a_{2}\right)>\frac{3}{4}$, implying $a_{2}>\frac{3}{2}-a_{1} \geq$ $\frac{3}{2}-\frac{13}{40}=\frac{47}{40}>1$, which is a contradiction. Hence, we can assume that

$$
a_{1} \leq E\left(X: X \in J_{1}\right)=\frac{1}{4}, \quad \text { and } \quad a_{2} \leq E\left(X: X \in J_{2}\right)=\frac{7}{8} .
$$

Suppose that $a_{2}<\frac{5}{8}$. Then,

$$
V_{2}>\frac{1}{4} \int_{\frac{3}{4}}^{1} 4\left(x-\frac{5}{8}\right)^{2} \mathrm{~d} x=\frac{13}{768}=0.0169271 \geq V_{2},
$$

which leads to a contradiction. So, we can assume that $\frac{5}{8} \leq a_{2}$. Thus, by (5), we have $\frac{5}{8} \leq a_{2} \leq \frac{7}{8}$. Assume that $\frac{5}{8} \leq a_{2} \leq \frac{3}{4}$. Since $a_{1} \leq \frac{1}{4}$, the following cases can arise:

Case 1. $\frac{1}{8} \leq a_{1} \leq \frac{1}{4}$.

Then, notice that $\frac{13}{32}<\frac{1}{2}\left(\frac{1}{4}+\frac{5}{8}\right)=\frac{7}{16}<\frac{1}{2}$, and so,

$$
\int_{\left[0, \frac{13}{32}\right]} \min _{a \in\left\{a_{1}, a_{2}\right\}}(x-a)^{2} \mathrm{~d} P=\frac{13\left(3072 a_{1}^{2}-1248 a_{1}+169\right)}{65536},
$$

the minimum value of which is $\frac{2197}{262144}$, and it occurs when $a_{1}=\frac{13}{64}$.

Notice that for $a_{1}=\frac{13}{64}$, we have

Thus, we have

$$
\frac{13}{32}=0.40625<\frac{1}{2}\left(\frac{13}{64}+\frac{5}{8}\right)=0.414063 .
$$

$$
\begin{aligned}
V_{2} \geq \frac{2197}{262144}+\frac{3}{4} \int_{\frac{13}{32}}^{\frac{7}{16}} 2\left(x-\frac{1}{4}\right)^{2} \mathrm{~d} x+\frac{3}{4} \int_{\frac{7}{16}}^{\frac{1}{2}} 2\left(x-\frac{5}{8}\right)^{2} \mathrm{~d} x+ \\
\frac{1}{4} \int_{\frac{3}{4}}^{1} 4\left(x-\frac{3}{4}\right)^{2} \mathrm{~d} x=\frac{13603}{786432},
\end{aligned}
$$

yielding $V_{2} \geq 0.0172971>V_{2}$, which is a contradiction.

Case 2. $a_{1}<\frac{1}{8}$.

Then, $\frac{1}{2}\left(\frac{1}{8}+\frac{5}{8}\right)=\frac{3}{8}<\frac{1}{2}$, and so 


$$
\begin{aligned}
V_{3} \geq \frac{3}{4} \int_{\frac{1}{8}}^{\frac{3}{8}} 2\left(x-\frac{1}{8}\right)^{2} \mathrm{~d} x+ & \frac{3}{4} \int_{\frac{3}{8}}^{\frac{1}{2}} 2\left(x-\frac{5}{8}\right)^{2} \mathrm{~d} x+ \\
& \frac{1}{4} \int_{\frac{3}{4}}^{1} 4\left(x-\frac{3}{4}\right)^{2} \mathrm{~d} x=\frac{61}{3072}=0.0198568>V_{3},
\end{aligned}
$$

which leads to a contradiction.

Hence, by Case 1 and Case 2, we can conclude that $\frac{3}{4} \leq a_{2} \leq \frac{7}{8}$. Suppose that $\frac{3}{4} \leq a_{2} \leq \frac{13}{16}$. Then, the Voronoi region of $a_{2}$ must contain points prom $J_{1}$ implying $\frac{1}{2}\left(a_{1}+a_{2}\right)<\frac{1}{2}$, which yields $a_{1}<1-a_{2} \leq 1-\frac{3}{4}=\frac{1}{4}$. Again,

$$
\int_{J_{1}}\left(x-a_{1}\right)^{2} \mathrm{~d} P=\frac{1}{16}\left(12 a^{2}-6 a+1\right),
$$

the minimum value of which is $\frac{1}{64}$ when $a_{1}=\frac{1}{4}$. Thus, we have

$$
V_{2} \geq \int_{J_{1}}\left(x-\frac{1}{4}\right)^{2} \mathrm{~d} P+\int_{J_{2}}\left(x-\frac{13}{16}\right)^{2} \mathrm{~d} P=\frac{55}{3072}=0.0179036>V_{2},
$$

which gives a contradiction. Hence, we can assume that $\frac{13}{16}<a_{2} \leq \frac{7}{8}$. Suppose that the Voronoi region of $a_{2}$ contains points from $J_{1}$, i.e., $\frac{1}{2}\left(a_{1}+a_{2}\right)<\frac{1}{2}$. Then, $a_{1}<1-a_{2} \leq 1-\frac{13}{16}=\frac{3}{16}$. Notice that

$$
\int_{J_{1}}\left(x-a_{1}\right)^{2} \mathrm{~d} P=\frac{1}{16}\left(12 a_{1}^{2}-6 a_{1}+1\right),
$$

the minimum value of which is $\frac{19}{1024}$ when $a_{1}=\frac{3}{16}$. Thus, we have $V_{2} \geq \frac{19}{1024}=$ $0.0185547>V_{2}$, which is a contradiction. Thus, we can assume that the Voronoi region of $a_{2}$ does not contain any point from $J_{1}$. Previously, we have proved that the Voronoi region of $a_{1}$ does not contain any point from $J_{2}$. Hence, we have

$$
a_{1}=E\left(X: X \in J_{1}\right)=\frac{1}{4}, \quad \text { and } \quad a_{2}=E\left(X: X \in J_{2}\right)=\frac{7}{8},
$$

and the corresponding quantization error for two-means is given by $V_{2}=\frac{13}{768}$.

LEMma 3.4. The set $\left\{\frac{1}{8}, \frac{3}{8}, \frac{7}{8}\right\}$ forms an optimal set of three-means with quantization error $V_{3}=\frac{1}{192}$.

Proof. Consider the set of three points $\beta$, such that $\beta:=\left\{\frac{1}{8}, \frac{3}{8}, \frac{7}{8}\right\}$. The distortion error due to the set $\beta$ is given by

$$
\int \min _{a \in \beta}(x-a)^{2} \mathrm{~d} P=2 \cdot \frac{3}{4} \int_{0}^{\frac{1}{4}} 2\left(x-\frac{1}{8}\right)^{2} \mathrm{~d} x+\frac{1}{4} \int_{\frac{3}{4}}^{1} 4\left(x-\frac{7}{8}\right)^{2} \mathrm{~d} x=\frac{1}{192} .
$$




\section{QUANTIZATION FOR A MIXTURE OF UNIFORM DISTRIBUTIONS}

Since $V_{3}$ is the quantization error for three-means, $V_{3} \leq \frac{1}{192}=0.00520833$. Let $\alpha:=\left\{a_{1}, a_{2}, a_{3}\right\}$ be an optimal set of three-means. Since the points in an optimal set are the conditional expectations in their own Voronoi regions, without any loss of generality, we can assume that $0<a_{1}<a_{2}<a_{3}<1$. We now show that $a_{2}<\frac{1}{2}$, and $\frac{3}{4}<a_{3}$. If $a_{3}<\frac{3}{4}$, then

$$
V_{3}>\int_{J_{2}}\left(x-\frac{3}{4}\right)^{2} \mathrm{~d} P=\frac{1}{4} \int_{\frac{3}{4}}^{1} 4\left(x-\frac{3}{4}\right)^{2} \mathrm{~d} x=\frac{1}{192}=0.00520833 \geq V_{3},
$$

which leads to a contradiction. Hence, we can assume that $\frac{3}{4}<a_{3}$. Next, we show that $a_{2}<\frac{1}{2}$. Suppose that $\frac{1}{2} \leq a_{2}$. Then,

$$
\begin{aligned}
& \int_{J_{1}} \min _{a \in\left\{a_{1}, \frac{1}{2}\right\}}(x-a)^{2} \mathrm{~d} P \\
& =\frac{3}{4} \int_{0}^{\frac{1}{2}\left(a_{1}+\frac{1}{2}\right)} 2\left(x-a_{1}\right)^{2} \mathrm{~d} x+\frac{3}{4} \int_{\frac{1}{2}\left(a_{1}+\frac{1}{2}\right)}^{\frac{1}{2}} 2\left(x-\frac{1}{2}\right)^{2} \mathrm{~d} x \\
& =\frac{1}{64}\left(24 a_{1}^{3}+12 a_{1}^{2}-6 a_{1}+1\right),
\end{aligned}
$$

the minimum value of which is $\frac{1}{144}$, and it occurs when $a_{1}=\frac{1}{6}$. Thus, in this case, we see that $V_{3} \geq \frac{1}{144}=0.00694444>V_{3}$, which leads to a contradiction. Hence, we can assume that $0<a_{1}<a_{2}<\frac{1}{2}$. Suppose that the Voronoi region of $a_{2}$ contains points from $J_{2}$. Then, $\frac{1}{2}\left(a_{2}+a_{3}\right)>\frac{3}{4}$ implying $a_{3}>\frac{3}{2}-a_{1} \geq \frac{3}{2}-\frac{1}{2}=1$, which is a contradiction, as $a_{3}<1$. Thus, we see that the Voronoi region of $a_{2}$ does not contain any point from $J_{2}$. Suppose that the Voronoi region of $a_{3}$ contains points from $J_{1}$. Then, $\frac{1}{2}\left(a_{2}+a_{3}\right)<\frac{1}{2}$ implying $a_{2}<1-a_{3} \leq 1-\frac{3}{4}=\frac{1}{4}$, and so

$$
V_{3}>\frac{3}{4} \int_{\frac{1}{4}}^{\frac{1}{2}} 2\left(x-\frac{1}{4}\right)^{2} \mathrm{~d} x=\frac{1}{128}=0.0078125>V_{3},
$$

which is a contradiction. So, we can assume that the Voronoi region of $a_{3}$ does not contain any point from $J_{1}$. Thus, by Theorem 1.2 , we can conclude that $a_{1}=\frac{1}{8}, a_{2}=\frac{3}{8}$, and $a_{3}=\frac{7}{8}$, and

$$
V_{3}=\int \min _{a \in \alpha}(x-a)^{2} \mathrm{~d} P=\frac{1}{192},
$$

which completes the proof of the lemma.

REMARK 3.5. By Lemma 3.3, and Lemma 3.4, we see that $\alpha_{2}=\alpha_{1}\left(P_{1}\right) \cup$ $\alpha_{1}\left(P_{2}\right)$, and $\alpha_{3}=\alpha_{2}\left(P_{1}\right) \cup \alpha_{1}\left(P_{2}\right)$. Using the similar technique, we can show that $\alpha_{4}=\alpha_{3}\left(P_{1}\right) \cup \alpha_{1}\left(P_{2}\right), \alpha_{5}=\alpha_{3}\left(P_{1}\right) \cup \alpha_{2}\left(P_{2}\right), \alpha_{6}=\alpha_{4}\left(P_{1}\right) \cup \alpha_{2}\left(P_{2}\right)$, $\alpha_{7}=\alpha_{5}\left(P_{1}\right) \cup \alpha_{2}\left(P_{2}\right), \alpha_{8}=\alpha_{6}\left(P_{1}\right) \cup \alpha_{2}\left(P_{2}\right)$, and $\alpha_{9}=\alpha_{6}\left(P_{1}\right) \cup \alpha_{3}\left(P_{2}\right)$. 


\section{MRINAL KANTI ROYCHOWDHURY — WASIELA SALINAS}

We now prove the following propositions.

Proposition 3.6. Let $\alpha_{n}$ be an optimal set of $n$-means for $P$ for $n \geq 2$. Then, the set $\alpha_{n}$ does not contain any point from the open interval $\left(\frac{1}{2}, \frac{3}{4}\right)$.

Pr o of. By Remark 3.5, the proposition is true for $2 \leq n \leq 9$. We now prove that the proposition is true for any positive integer $n \geq 10$. Take any $n \geq 10$. Since $\alpha_{9}=\alpha_{6}\left(P_{1}\right) \cup \alpha_{3}\left(P_{2}\right)$, and the Voronoi region of any point in $\alpha_{9} \cap J_{1}$ does not contain any point from $J_{2}$, and the Voronoi region of any point in $\alpha_{9} \cap J_{2}$ does not contain any point from $J_{1}$, we have

$$
V_{9}=\frac{3}{4} V_{6}\left(P_{1}\right)+\frac{1}{4} V_{3}\left(P_{2}\right)=\frac{1}{1728}=0.000578704 .
$$

Since $V_{n}$ is the quantization error for $n$-means for $n \geq 10$, we have $V_{n} \leq V_{9}=$ 0.000578704 . Let $\alpha_{n}:=\left\{a_{1}, a_{2}, \ldots, a_{n}\right\}$ be an optimal set of $n$-means for $P$ such that $a_{1}<a_{2}<\cdots<a_{n}$. Let $j=\max \left\{i: a_{i} \leq \frac{1}{2}\right\}$. Then, $a_{j} \leq \frac{1}{2}<a_{j+1}$. The proposition will be proved if we can show that $a_{j+1} \in J_{2}$. For the sake of contradiction, assume that $a_{j+1} \in\left(\frac{1}{2}, \frac{3}{4}\right)$. Then, the following two cases can arise:

Case 1. $\frac{1}{2}<a_{j+1} \leq \frac{5}{8}$

In this case, the Voronoi region of $a_{j+1}$ must contain points from $J_{2}$, otherwise, the quantization error can be strictly reduced my moving the point $a_{j+1}$ to $\frac{1}{2}$. Thus, $\frac{1}{2}\left(a_{j+1}+a_{j+2}\right)>\frac{3}{4}$ implying $a_{j+2}>\frac{3}{2}-a_{j+1} \geq \frac{3}{2}-\frac{5}{8}=\frac{7}{8}$, which yields the fact that

$$
V_{n} \geq \int_{\left[\frac{3}{4}, \frac{7}{8}\right]}\left(x-\frac{7}{8}\right)^{2} \mathrm{~d} P=\frac{1}{4} \int_{\frac{3}{4}}^{\frac{7}{8}} 4\left(x-\frac{7}{8}\right)^{2} \mathrm{~d} x=0.000651042>V_{n},
$$

which leads to a contradiction.

Case 2. $\frac{5}{8} \leq a_{j+1}<\frac{3}{4}$.

In this case, we have $\frac{1}{2}\left(a_{j}+a_{j+1}\right)<\frac{1}{2}$ implying $a_{j}<1-a_{j+1} \leq 1-\frac{5}{8}=\frac{3}{8}$, which yields the fact that

$$
V_{n} \geq \int_{\left[\frac{3}{8}, \frac{1}{2}\right]}\left(x-\frac{3}{8}\right)^{2} \mathrm{~d} P=\frac{3}{4} \int_{\frac{3}{8}}^{\frac{1}{2}} 2\left(x-\frac{3}{8}\right)^{2} \mathrm{~d} x=0.000976563>V_{n},
$$

which is a contradiction.

In light of the above two cases, we can conclude that $a_{j+1} \notin\left(\frac{1}{2}, \frac{3}{4}\right)$. Hence, $\frac{3}{4}<a_{j+2}$, i.e., $a_{j+2} \in J_{2}$. Thus, the proof of the proposition is complete. 
Proposition 3.7. Let $\alpha_{n}$ be an optimal set of $n$-means for $P$ for $n \geq 2$. Then, for $n \geq 2, \alpha_{n} \cap J_{1} \neq \emptyset$, and $\alpha_{n} \cap J_{2} \neq \emptyset$. Moreover, for $n \geq 2$, any point in $\alpha_{n} \cap J_{1}$ does not contain any point from $J_{2}$, and any point in $\alpha_{n} \cap J_{2}$ does not contain any point from $J_{1}$,

Proof. As shown in the proof of Lemma 3.3. and Lemma 3.4, we see that the proposition is true for $n=2,3$. By Lemma 3.4 we know $V_{3}=\frac{1}{192}=$ 0.00520833 . We now prove the proposition for $n \geq 4$. Let $n \geq 4$. Since $V_{n}$ is the quantization error for $n$-means for $n \geq 4$, we have $V_{n} \leq V_{3}=0.00520833$. Let $\alpha_{n}:=\left\{a_{1}, a_{2}, \ldots, a_{n}\right\}$ be an optimal set of $n$-means for $P$ such that $a_{1}<a_{2}<\cdots<a_{n}$. If $\alpha_{n} \cap J_{2}=\emptyset$, then

$$
V_{n}>\frac{1}{4} \int_{\frac{3}{4}}^{1} 4\left(x-\frac{3}{4}\right)^{2} \mathrm{~d} x=0.00520833
$$

which is a contradiction as $V_{n} \leq 0.00520833$. On the other hand, if $\alpha_{n} \cap J_{1}=\emptyset$, then

$$
V_{n}>\frac{3}{4} \int_{0}^{\frac{1}{2}} 2\left(x-\frac{1}{4}\right)^{2} \mathrm{~d} x=\frac{1}{64}=0.015625>V_{n},
$$

which leads to a contradiction. Hence,

$$
\alpha_{n} \cap J_{1} \neq \emptyset, \quad \text { and } \quad \alpha_{n} \cap J_{2} \neq \emptyset \text {. }
$$

Let $j=\max \left\{i: a_{i} \leq \frac{1}{2}\right\}$. Then, $a_{j} \leq \frac{1}{2}$, and due to Proposition 3.6, we have $\frac{3}{4} \leq a_{j+1}$. If the Voronoi region of $a_{j}$ contains points from $J_{2}$, then $\frac{1}{2}\left(a_{j}+\right.$ $\left.a_{j+1}\right)>\frac{3}{4}$ implying $a_{j+1}>\frac{3}{2}-a_{j} \geq \frac{3}{2}-\frac{1}{2}=1$, which is a contradiction. If the Voronoi region of $a_{j+1}$ contains points from $J_{1}$, then $\frac{1}{2}\left(a_{j}+a_{j+1}\right)<\frac{1}{2}$ implying $a_{j}<1-a_{j+1} \leq 1-\frac{3}{4}=\frac{1}{4}$. Then,

$$
V_{n} \geq \int_{\left[\frac{1}{4}, \frac{1}{2}\right]}\left(x-\frac{1}{4}\right)^{2} \mathrm{~d} P=\frac{3}{4} \int_{\frac{1}{4}}^{\frac{1}{2}} 2\left(x-\frac{1}{4}\right)^{2} \mathrm{~d} x=\frac{1}{128}
$$

yielding $V_{n} \geq 0.0078125>V_{n}$, which leads to a contradiction. Thus, the proof of the proposition is complete.

Definition 3.8. For $n \in \mathbb{N}$, and $n \geq 2$, define the function $a(n)$ as follows:

$$
a(n)=\min \{k \in \mathbb{N}: H(n, k)>0\},
$$

where $H(n, k)=\frac{1}{n^{3}}-\sum_{i=k}^{\infty} \frac{1}{(i+1)^{4}}$.

REMARK 3.9. Notice that $\sum_{i=k}^{\infty} \frac{1}{(i+1)^{4}}$ is a decreasing function of $k \in \mathbb{N}$, and so for a given $n \geq 2, H(n, k)$ is an increasing function of $k$, and thus the function $a(n)$ is well defined. Moreover, $\left\{\frac{1}{n^{3}}\right\}_{n \geq 2}$ is a decreasing sequence, and so, 
the sequence $\{a(n)\}_{n=2}^{\infty}$ is an increasing sequence. In fact,

$$
\{a(n)\}_{n=2}^{\infty}=\{1,2,3,3,4,5,6,6,7,8,8,9,10,10,
$$

$11,12,12,13,14,15,15,16,17,17,18, \ldots\}$.

By $\lfloor x\rfloor$ it is meant the greatest integer not exceeding $x$. To find the value of $a(n)$ for any positive integer $n$, one can start checking by putting $k=\left\lfloor\frac{2 n}{3}\right\rfloor$ in the function $H(n, k)$. If $H(n, k)>0$, then find $H(n, k-1), H(n, k-2), \ldots$ until one obtains some positive integer $m$, such that $H(n, m)>0$, and $H(n, m-1)<0$, and then $a(n)=m$. If $H(n, k)<0$, then find $H(n, k+1), H(n, k+2), \ldots$ until one obtains some positive integer $m$, such that $H(n, m)>0$, and $H(n, m-1)<0$, and then $a(n)=m$.

REMARK 3.10. For $n \geq 2$ let $\alpha_{n}$ be an optimal set of $n$-means for $P$. Due to Proposition 3.6 and Proposition 3.7, we can conclude that if $\alpha_{n}$ contains $k$ elements from $J_{1}$, then $\alpha_{n}$ contains $n-k$ elements from $J_{2}$. Thus, we have

$$
\begin{aligned}
V_{n}:=V_{n}(P) & =\int \min _{a \in \alpha_{n}}(x-a)^{2} \mathrm{~d} P \\
& =\frac{3}{4} \int \min _{a \in \alpha_{n} \cap J_{1}}(x-a)^{2} \mathrm{~d} P_{1}+\frac{1}{4} \int \min _{a \in \alpha_{n} \cap J_{2}}(x-a)^{2} \mathrm{~d} P_{2},
\end{aligned}
$$

yielding

$$
V_{n}(P)=\frac{3}{4} V_{k}\left(P_{1}\right)+\frac{1}{4} V_{n-k}\left(P_{2}\right) .
$$

Let us now give the following theorem, which gives the optimal sets of $n$-means and the $n$th quantization errors for the mixed distribution $P$ for all positive integers $n \geq 2$.

TheOREM 3.11. For $n \geq 2$, let $\alpha_{n}$ be an optimal set of $n$-means for $P$. Then, $\alpha_{n}$ contains $a(n)$ elements from $J_{1}$, i.e.,

$$
\alpha_{n}(P)=\alpha_{a(n)}\left(P_{1}\right) \cup \alpha_{n-a(n)}\left(P_{2}\right), \text { and } V_{n}(P)=\frac{3}{4} V_{a(n)}\left(P_{1}\right)+\frac{1}{4} V_{n-a(n)}\left(P_{2}\right) \text {. }
$$

Proof. Assume that $\alpha_{n}$ contains $k$ elements from $J_{1}$. Let $V(k, n-k)$ is the corresponding distortion error. Then, as mentioned in Remark 3.10, we have

$$
V(k, n-k)=\frac{3}{4} V_{k}\left(P_{1}\right)+\frac{1}{4} V_{n-k}\left(P_{2}\right) .
$$

Notice that if our assumption is correct, then we must have $V_{n}=V(k, n-k)$. 
Let us now run the following algorithm:

(i) Write $k:=\left\lfloor\frac{2 n}{3}\right\rfloor$.

(ii) If $V(k-1, n-(k-1))<V(k, n-k)$ replace $k$ by $k-1$ and return, else go to step (iii).

(iii) If $V(k+1, n-(k+1))<V(k, n-k)$ replace $k$ by $k+1$ and return, else step (iv).

(iv) End.

After running the above algorithm, we see that $k=a(n)$, i.e., our assumption is correct. Thus, the proof of the theorem is complete.

REMARK 3.12. If $n=14$, then $k=\left\lfloor\frac{28}{3}\right\rfloor=9$. By running the algorithm as mentioned in the theorem, we obtain $k=10$. Moreover, notice that $a(14)=10$, i.e., $\alpha_{14}$ contains $a(14)$ elements from $J_{1}$, and $n-a(14)$ elements from $J_{2}$, i.e., $\alpha_{14}=\alpha_{a(14)}\left(P_{1}\right) \cup \alpha_{14-a(14)}\left(P_{2}\right)$. If $n=100$, then $k=\left\lfloor\frac{200}{3}\right\rfloor=66$. By running the algorithm as mentioned in the theorem, we obtain $k=69$. Moreover, we have $a(100)=69$, i.e., $\alpha_{100}$ contains $a(100)$ elements from $J_{1}$, and $n-a(100)$ elements from $J_{2}$, i.e., $\alpha_{100}=\alpha_{a(100)}\left(P_{1}\right) \cup \alpha_{100-a(100)}\left(P_{2}\right)$.

\section{Optimal quantization for the mixture of two uniform distributions on two connected line segments}

Let $P_{1}$ and $P_{2}$ be two uniform distributions, respectively, on the intervals $\left[0, \frac{1}{2}\right]$ and $\left[\frac{1}{2}, 1\right]$. Write

$$
J_{1}:=\left[0, \frac{1}{2}\right], \quad \text { and } \quad J_{2}:=\left[\frac{1}{2}, 1\right] .
$$

Let $f_{1}$ and $f_{2}$ be their respective density functions. Then, $f_{1}(x)=2$ if $x \in\left[0, \frac{1}{2}\right]$, and zero, otherwise; and $f_{2}(x)=2$ if $x \in\left[\frac{1}{2}, 1\right]$, and zero, otherwise. Let $P:=\frac{3}{4} P_{1}+\frac{1}{4} P_{2}$. For such a mixed distribution, in this section, we investigate the optimal sets of $n$-means and the $n$th quantization errors for all $n \in \mathbb{N}$. Notice that the density function of the mixed distribution $P$ can be written as follows:

$$
f(x)=\left\{\begin{array}{cc}
\frac{3}{2} & \text { if } x \in J_{1} \\
\frac{1}{2} & \text { if } x \in J_{2}, \\
0, & \text { otherwise. }
\end{array}\right.
$$




\section{MRINAL KANTI ROYCHOWDHURY — WASIELA SALINAS}

Let us now prove the following lemma.

Lemma 4.1. Let $P$ be the mixed distribution defined by $P=\frac{3}{4} P_{1}+\frac{1}{4} P_{2}$. Then, $E(P)=\frac{3}{8}$, and $V(P)=\frac{13}{192}$.

P r o of. We have

$E(P)=\int x \mathrm{~d} P=\frac{3}{4} \int x \mathrm{~d}\left(P_{1}(x)\right)+\frac{1}{4} \int x \mathrm{~d}\left(P_{2}(x)\right)=\frac{3}{4} \int_{0}^{\frac{1}{2}} 2 x \mathrm{~d} x+\frac{1}{4} \int_{\frac{1}{2}}^{1} 2 x \mathrm{~d} x$

yielding $E(P)=\frac{3}{8}$, and

$V(P)=\int(x-E(P))^{2} \mathrm{~d} P=\frac{3}{4} \int(x-E(P))^{2} \mathrm{~d}\left(P_{1}(x)\right)+\frac{1}{4} \int(x-E(P))^{2} \mathrm{~d}\left(P_{2}(x)\right)$, implying $V(P)=\frac{13}{192}$, and thus, the lemma is yielded.

REMARK 4.2. The optimal set of one-mean is the set $\left\{\frac{3}{8}\right\}$, and the corresponding quantization error is the variance $V:=V(P)$ of a random variable with distribution $P$.

Proposition 4.3. For $n \geq 2$, let $\alpha_{n}$ be an optimal set of $n$-means. Then, $\alpha_{n} \cap J_{1} \neq \emptyset$, and $\alpha_{n} \cap J_{2} \neq \emptyset$.

P r o of. Consider the set of two points $\beta:=\left\{\frac{1}{4}, \frac{3}{4}\right\}$. The distortion error due to the set $\beta$ is given by

$$
\begin{aligned}
\int \min _{b \in \beta}(x-b)^{2} \mathrm{~d} P & =\int_{J_{1}}\left(x-\frac{1}{4}\right)^{2} \mathrm{~d} P+\int_{J_{2}}\left(x-\frac{3}{4}\right)^{2} \mathrm{~d} P \\
& =\frac{3}{4} \int_{0}^{\frac{1}{2}} 2\left(x-\frac{1}{4}\right)^{2} \mathrm{~d} x+\frac{1}{4} \int_{\frac{1}{2}}^{1} 2\left(x-\frac{3}{4}\right)^{2} \mathrm{~d} x=\frac{1}{48} .
\end{aligned}
$$

Since $V_{n}$ is the quantization error for two-means, and $n \geq 2$, we have $V_{n} \leq V_{2} \leq$ $\frac{1}{48}=0.0208333$. For the sake of contradiction assume that $\alpha_{n} \cap J_{2}=\emptyset$. Then,

$$
V_{n}>\int_{J_{2}}\left(x-\frac{1}{2}\right)^{2} \mathrm{~d} P=\frac{1}{4} \int_{\frac{1}{2}}^{1} 2\left(x-\frac{1}{2}\right)^{2} \mathrm{~d} x=\frac{1}{48} \geq V_{n},
$$

which is a contradiction. Hence, we can assume that $\alpha \cap J_{2} \neq \emptyset$. Similarly, we can show that $\alpha_{n} \cap J_{1} \neq \emptyset$. Thus, the proof of the proposition is complete.

LEMMA 4.4. The set $\left\{\frac{1}{4}, \frac{3}{4}\right\}$ forms an optimal set of two-means with quantization error $V_{2}=\frac{1}{48}$. 


\section{QUANTIZATION FOR A MIXTURE OF UNIFORM DISTRIBUTIONS}

P r o o f. Let $\alpha:=\left\{a_{1}, a_{2}\right\}$ be an optimal set of two-means such that $0<a_{1}<$ $a_{2}<1$. By Proposition 4.3, we have $a_{1}<\frac{1}{2}<a_{2}$. The following two cases can arise:

Case 1. $\frac{1}{2} \leq \frac{a_{1}+a_{2}}{2}$.

In this case, we have

$$
a_{1}=\frac{\frac{3}{4} \int_{0}^{\frac{1}{2}} 2 x \mathrm{~d} x+\frac{1}{4} \int_{\frac{1}{2}}^{\frac{1}{2}\left(a_{1}+a_{2}\right)} 2 x \mathrm{~d} x}{\frac{3}{4} \int_{0}^{\frac{1}{2}} 2 \mathrm{~d} x+\frac{1}{4} \int_{\frac{1}{2}}^{\frac{1}{2}\left(a_{1}+a_{2}\right)} 2 \mathrm{~d} x}, \quad \text { and } \quad a_{2}=\frac{1}{2}\left(\frac{1}{2}\left(a_{1}+a_{2}\right)+1\right) .
$$

Solving the above two equations, we have $a_{1}=\frac{1}{4}$, and $a_{2}=\frac{3}{4}$, with distortion error

$$
\begin{aligned}
V(P ; \alpha)= & \frac{3}{4} \int_{0}^{\frac{1}{2}} 2\left(x-a_{1}\right)^{2} \mathrm{~d} x \\
& +\frac{1}{4} \int_{\frac{1}{2}}^{\frac{1}{2}\left(a_{1}+a_{2}\right)} 2\left(x-a_{1}\right)^{2} \mathrm{~d} x+\frac{1}{4} \int_{\frac{1}{2}\left(a_{1}+a_{2}\right)}^{1} 2\left(x-a_{2}\right)^{2} \mathrm{~d} x=\frac{1}{48} .
\end{aligned}
$$

Case 2. $\frac{a_{1}+a_{2}}{2}<\frac{1}{2}$.

Proceeding in the similar way as Case 1, we obtain two equations, and see that there is no solution in this case.

Considering the above two cases, we see that the set $\left\{\frac{1}{4}, \frac{3}{4}\right\}$ forms an optimal set of two-means with quantization error $\frac{1}{48}$, which is the lemma.

LEMMA 4.5. The set

$$
\left\{\frac{1}{3}\left(\frac{1}{8}(21-\sqrt{3})-2\right), \frac{1}{8}(21-\sqrt{3})-2, \frac{1}{24}(21-\sqrt{3})\right\}
$$

forms an optimal set of three-means with quantization error $V_{3}=0.00787482$.

Proof. Consider the set of three points $\beta:=\{u, v, w\}$, where

$$
u=\frac{1}{3}\left(\frac{1}{8}\right)(21-\sqrt{3})-2, \quad v=\frac{1}{8}(21-\sqrt{3})-2, \quad \text { and } \quad w=\frac{1}{24}(21-\sqrt{3}) .
$$

Since $0<u<v<\frac{1}{2}<\frac{v+w}{2}<w<1$, the distortion error due to the set $\beta$ is given by

$$
\begin{aligned}
V(P ; \beta)= & \frac{3}{4} \int_{0}^{\frac{u+v}{2}} 2(x-u)^{2} \mathrm{~d} x+\frac{3}{4} \int_{\frac{u+v}{2}}^{\frac{1}{2}} 2(x-v)^{2} \mathrm{~d} x \\
& +\frac{1}{4} \int_{\frac{1}{2}}^{\frac{v+w}{2}} 2(x-v)^{2} \mathrm{~d} x+\frac{1}{4} \int_{\frac{v+w}{2}}^{1} 2(x-w)^{2} \mathrm{~d} x
\end{aligned}
$$


yielding $V(P ; \beta)=0.00787482$. Since $V_{3}$ is the quantization error for threemeans we have $V_{3} \leq 0.00787482$. Let $\alpha:=\{a, b, c\}$ be an optimal set of threemeans. Without any loss of generality we can assume that $0<a<b<c<1$. By Proposition 4.3, we know $a<\frac{1}{2}<c$. We now show that $b<\frac{1}{2}$. Suppose that $\frac{9}{16}<b$. Then,

$$
\begin{aligned}
V_{3} & \geq \int_{J_{1}} \min _{r \in\left\{a, \frac{9}{16}\right\}}(x-r)^{2} \mathrm{~d} P \\
& =\frac{3}{4} \int_{0}^{\frac{1}{2}\left(a+\frac{9}{16}\right)} 2(x-a)^{2} \mathrm{~d} x+\frac{3}{4} \int_{\frac{1}{2}\left(a+\frac{9}{16}\right)}^{\frac{1}{2}} 2\left(x-\frac{9}{16}\right)^{2} \mathrm{~d} x \\
& =\frac{12288 a^{3}+6912 a^{2}-3888 a+725}{32768},
\end{aligned}
$$

the minimum value of which is 0.00976563 and it occurs when $a=\frac{3}{16}$, and thus, we have $V_{3} \geq 0.00976563>V_{3}$, which is a contradiction. So, we can assume that $b \leq \frac{9}{16}$. Next, assume that $\frac{1}{2} \leq b \leq \frac{9}{16}$. Notice that then $\frac{9}{16}<c<1$. Then, as before we have

$$
\begin{aligned}
V_{3} & \geq \int_{J_{1}} \min _{r \in\left\{a, \frac{1}{2}\right\}}(x-r)^{2} \mathrm{~d} P+\int_{\frac{9}{16}}^{1} \min _{s \in\left\{\frac{9}{16}, c\right\}}(x-r)^{2} \mathrm{~d} P \\
& =\frac{1}{64}\left(24 a^{3}+12 a^{2}-6 a+1\right)+\frac{-12288 c^{3}+42240 c^{2}-45264 c+15655}{98304},
\end{aligned}
$$

the minimum value of which is $\frac{1}{144}+\frac{343}{221184}=0.00849519$, and it occurs when $a=0.166667$, and $c=0.854167$. Thus, we have $V_{3} \geq 0.00849519>V_{3}$, which is a contradiction. Hence, we can assume that $b<\frac{1}{2}$. Then, the two cases can arise: either $\frac{1}{2}(b+c)<\frac{1}{2}$, or $\frac{1}{2} \leq \frac{1}{2}(b+c)$. Proceeding as in Lemma 4.4, we can see that $\frac{1}{2}(b+c)<\frac{1}{2}$ can not happen. Thus, we have $\frac{1}{2} \leq \frac{1}{2}(b+c)$ implying

$$
a=\frac{a+b}{4}, \quad b=\frac{\frac{3}{4} \int_{\frac{a+b}{2}}^{\frac{1}{2}} 2 x \mathrm{~d} x+\frac{1}{4} \int_{\frac{1}{2}}^{\frac{b+c}{2}} 2 x \mathrm{~d} x}{\frac{3}{4} \int_{\frac{a+b}{2}}^{\frac{1}{2}} 2 \mathrm{~d} x+\frac{1}{4} \int_{\frac{1}{2}}^{\frac{b+c}{2}} 2 \mathrm{~d} x}, \quad \text { and } \quad c=\frac{\int_{\frac{b+c}{2}}^{1} 2 x \mathrm{~d} x}{\frac{4}{4} \int_{\frac{b+c}{2}}^{1} 2 \mathrm{~d} x} .
$$

Solving the above equations, we have

$$
a=\frac{1}{3}\left(\frac{1}{8}(21-\sqrt{3})-2\right), \quad b=\frac{1}{8}(21-\sqrt{3})-2, \quad \text { and } \quad c=\frac{1}{24}(21-\sqrt{3}),
$$

and the corresponding quantization error is given by $V_{3}=0.00787482$, and thus, the proof of the lemma is complete. 
Definition 4.6. For $n \in \mathbb{N}$, define the sequence $\{a(n)\}_{n=1}^{\infty}$ as follows:

i.e.,

$$
a(n):=\left\lfloor\frac{5(n+1)}{8}\right\rfloor,
$$

$$
\{a(n)\}_{n=1}^{\infty}=\{1,1,2,3,3,4,5,5,6,6,7,8,8,9,10,10,
$$

$$
11,11,12,13,13,14,15,15,16,16, \ldots\} .
$$

Let us now state and prove the following two claims.

Claim 4.7. Let $\{a(n)\}$ be the sequence defined by Definition 4.6. Take $n=8$, and then $a(n)=5$. Assume that $\alpha_{n}:=\left\{a_{1}<a_{2}<a_{3}<a_{4}<a_{5}<b_{1}<b_{2}<b_{3}\right\}$ is an optimal set of eight-means for $P$. Then, $\frac{1}{2} \leq \frac{1}{2}\left(a_{5}+b_{1}\right)$.

Proof. For the sake of contradiction, assume that $\frac{1}{2}\left(a_{5}+b_{1}\right)<\frac{1}{2}$. Then,

$$
a_{1}=\frac{1}{2}\left(0+\frac{a_{1}+a_{2}}{2}\right), \quad \text { and } \quad a_{2}=\frac{1}{2}\left(\frac{a_{1}+a_{2}}{2}+\frac{a_{2}+a_{3}}{2}\right)
$$

implying $a_{1}=\frac{1}{3} a_{2}$, and $a_{2}=\frac{3}{5} a_{3}$. Similarly, $a_{3}=\frac{5}{7} a_{4}, a_{4}=\frac{7}{9} a_{5}$.

Again,

$$
b_{2}=\frac{1}{2}\left(\frac{b_{1}+b_{2}}{2}+\frac{b_{2}+b_{3}}{2}\right), \quad \text { and } \quad b_{3}=\frac{1}{2}\left(\frac{b_{2}+b_{3}}{2}+1\right)
$$

implying $b_{2}=\frac{3}{5} b_{1}+\frac{2}{5}$, and $b_{3}=\frac{1}{3} b_{2}+\frac{2}{3}$. Moreover,

$$
a_{5}=\frac{1}{2}\left(\frac{a_{4}+a_{5}}{2}+\frac{a_{5}+b_{1}}{2}\right)=\frac{1}{2}\left(\frac{\frac{7}{9} a_{5}+a_{5}}{2}+\frac{a_{5}+b_{1}}{2}\right)
$$

implying $a_{5}=\frac{9}{11} b_{1}$, and

$$
\begin{aligned}
b_{1} & =E\left(X: X \in\left[\frac{a_{5}+b_{1}}{2}, \frac{1}{2}\right] \cup\left[\frac{1}{2}, \frac{b_{1}+b_{2}}{2}\right]\right) \\
& =\frac{-6 a_{5} b_{1}-3 a_{5}^{2}-2 b_{1}^{2}+b_{2}^{2}+2 b_{1} b_{2}+2}{-12 a_{5}-8 b_{1}+4 b_{2}+8} .
\end{aligned}
$$

Next, putting the values of $a_{5}$ and $b_{2}$ in the expression of $b_{1}$, we have

$$
b_{1}=\frac{-11128 b_{1}^{2}+1936 b_{1}+3267}{14520-23320 b_{1}} \text { yielding } \quad b_{1}=\frac{11(143 \pm 5 i \sqrt{5})}{3048},
$$

which is not real. Thus, $\frac{1}{2}\left(a_{5}+b_{1}\right)<\frac{1}{2}$ leads to a contradiction. Hence, $\frac{1}{2} \leq \frac{1}{2}\left(a_{5}+b_{1}\right)$.

Claim 4.8. Let $\{a(n)\}$ be the sequence defined by Definition 4.6. Take $n=9$, and then $a(n)=6$. Assume that $\alpha_{n}:=\left\{a_{1}<a_{2}<a_{3}<a_{4}<a_{5}<a_{6}<b_{1}<\right.$ $\left.b_{2}<b_{3}\right\}$ is an optimal set of nine-means for $P$. Then, $\frac{1}{2} \leq \frac{1}{2}\left(a_{6}+b_{1}\right)$. 
Pr o of. For the sake of contradiction, assume that $\frac{1}{2}\left(a_{6}+b_{1}\right)<\frac{1}{2}$. Then,

$$
a_{1}=\frac{1}{2}\left(0+\frac{a_{1}+a_{2}}{2}\right), \quad \text { and } \quad a_{2}=\frac{1}{2}\left(\frac{a_{1}+a_{2}}{2}+\frac{a_{2}+a_{3}}{2}\right)
$$

implying $a_{1}=\frac{1}{3} a_{2}$, and $a_{2}=\frac{3}{5} a_{3}$. Similarly, $a_{3}=\frac{5}{7} a_{4}, a_{4}=\frac{7}{9} a_{5}$, and $a_{5}=\frac{9}{11} a_{6}$. Again,

$$
b_{2}=\frac{1}{2}\left(\frac{b_{1}+b_{2}}{2}+\frac{b_{2}+b_{3}}{2}\right), \quad \text { and } \quad b_{3}=\frac{1}{2}\left(\frac{b_{2}+b_{3}}{2}+1\right)
$$

implying $b_{2}=\frac{3}{5} b_{1}+\frac{2}{5}$, and $b_{3}=\frac{1}{3} b_{2}+\frac{2}{3}$. Moreover,

$a_{6}=\frac{1}{2}\left(\frac{a_{5}+a_{6}}{2}+\frac{a_{6}+b_{1}}{2}\right)=\frac{1}{2}\left(\frac{\frac{9}{11} a_{6}+a_{6}}{2}+\frac{a_{6}+b_{1}}{2}\right) \quad$ implying $a_{6}=\frac{11}{13} b_{1}$, and

$$
\begin{aligned}
b_{1} & =E\left(X: X \in\left[\frac{a_{6}+b_{1}}{2}, \frac{1}{2}\right] \cup\left[\frac{1}{2}, \frac{b_{1}+b_{2}}{2}\right]\right) \\
& =\frac{-6 a_{5} b_{1}-3 a_{5}^{2}-2 b_{1}^{2}+b_{2}^{2}+2 b_{1} b_{2}+2}{-12 a_{5}-8 b_{1}+4 b_{2}+8} .
\end{aligned}
$$

Next, putting the values of $a_{5}$ and $b_{2}$ in the expression of $b_{1}$, we have

$$
b_{1}=\frac{-16192 b_{1}^{2}+2704 b_{1}+4563}{20280-33280 b_{1}} \quad \text { yielding } \quad b_{1}=\frac{13(169 \pm 5 i \sqrt{11})}{4272},
$$

which is not real. Thus, $\frac{1}{2}\left(a_{6}+b_{1}\right)<\frac{1}{2}$ leads to a contradiction. Hence, $\frac{1}{2} \leq$ $\frac{1}{2}\left(a_{6}+b_{1}\right)$.

LEMMA 4.9. Let $\alpha_{n}$ be an optimal set of $n$-means for P $\check{n} 1$, where $n \geq 2$, and $\{a(n)\}$ be the sequence defined by Definition 4.6. Then, $\operatorname{card}\left(\alpha_{n} \cap J_{1}\right)=a(n)$, and $\operatorname{card}\left(\alpha_{n} \cap J_{2}\right)=n-a(n)$.

Proof. We prove the lemma by induction. By Lemma 4.4 and Lemma 4.5. the lemma is true for $n=2,3$. Assume that that the lemma is true for $n=\ell$, i.e., $\operatorname{card}\left(\alpha_{\ell} \cap J_{1}\right)=a(\ell)$, and $\operatorname{card}\left(\alpha_{\ell} \cap J_{2}\right)=n-a(\ell)$. We need to show that $\operatorname{card}\left(\alpha_{\ell+1} \cap J_{1}\right)=a(\ell+1)$. Assume that $\operatorname{card}\left(\alpha_{\ell+1} \cap J_{1}\right)=k$, i.e., $\alpha_{\ell+1}$ contains $k$ elements from $J_{1}$, and $n-k$ elements from $J_{2}$. Let

$\alpha_{\ell+1} \cap J_{1}=\left\{a_{1}<a_{2}<\cdots<a_{k}\right\}, \quad$ and $\quad \alpha_{\ell+1} \cap J_{2}=\left\{b_{1}<b_{2}<\cdots<b_{n-k}\right\}$.

Then, either $\frac{1}{2}\left(a_{k}+b_{1}\right)<\frac{1}{2}$, or $\frac{1}{2}<\frac{1}{2}\left(a_{k}+b_{1}\right)$. In each case, using the similar techniques as in the proofs of Claim 4.7 and Claim 4.8, if the solution exists, we solve for $a_{1}, a_{2}, \ldots, a_{k}, b_{1}, \ldots, b_{n-1}$, and find the distortion errors. Notice that at least one solution will exist. Let $V(k, n-k)$ be the minimum of the distortion errors if $\alpha_{\ell+1}$ contains $k$ elements from $J_{1}$, and $n-k$ elements from $J_{2}$. 


\section{QUANTIZATION FOR A MIXTURE OF UNIFORM DISTRIBUTIONS}

Let us now run the following algorithm:

(i) Write $k:=a(\ell)$.

(ii) If $V(k-1, n-(k-1))<V(k, n-k)$ replace $k$ by $k-1$ and return, else go to step (iii).

(iii) If $V(k+1, n-(k+1))<V(k, n-k)$ replace $k$ by $k+1$ and return, else step (iv).

(iv) End.

After running the above algorithm, we see that the value of $k$ obtained equals $a(\ell+1)$, i.e., the lemma is true for $n=\ell+1$ if it is true for $n=\ell$. Hence, by the Induction Principle, we can say that the lemma is true for all positive integers $n \geq 2$, i.e., $\operatorname{card}\left(\alpha_{n} \cap J_{1}\right)=a(n)$ for any positive integer $n \geq 2$. Since $\operatorname{card}\left(\alpha_{n} \cap J_{1}\right)+\operatorname{card}\left(\alpha_{n} \cap J_{2}\right)=n$, we have $\operatorname{card}\left(\alpha_{n} \cap J_{2}\right)=n-a(n)$. Thus, the proof of the lemma is complete.

Let us now state and prove the following theorem which is the main theorem in this section.

TheOREM 4.10. Let $\alpha_{n}$ be an optimal set of $n$-means for $P$, where $n \geq 2$, and $\{a(n)\}$ be the sequence defined by Definition 4.6. Write $k:=a(n), m:=n-a(n)$. Then,

where

$$
\alpha_{n}:=\left\{a_{1}<a_{2}<\cdots<a_{k}<b_{1}<b_{2}<\cdots<b_{m}\right\},
$$

$$
a_{j}=\left\{\begin{array}{clcrl}
\frac{a_{1}+a_{2}}{4} & \text { if } & j=1, \\
\frac{1}{2}\left(\frac{a_{j-1}+a_{j}}{2}+\frac{a_{j}+a_{j+1}}{2}\right) & \text { if } & 2 \leq j \leq k-1, \\
E\left(X: X \in\left[\frac{a_{k-1}+a_{k}}{2}, \frac{1}{2}\right] \cup\left[\frac{1}{2}, \frac{a_{k}+b_{1}}{2}\right]\right) & \text { if } & j=k,
\end{array}\right.
$$

and

$$
b_{j}=\left\{\begin{array}{clrl}
\frac{1}{2}\left(\frac{a_{k}+b_{1}}{2}+\frac{b_{1}+b_{2}}{2}\right) & \text { if } & j=1, \\
\frac{1}{2}\left(\frac{b_{j-1}+b_{j}}{2}+\frac{b_{j}+b_{j+1}}{2}\right) & \text { if } & 2 \leq j \leq m-1, \\
\frac{1}{2}\left(\frac{b_{m-1}+b_{m}}{2}+1\right) & \text { if } & j=m,
\end{array}\right.
$$

and the corresponding quantization error is given by

$$
\begin{gathered}
V_{n}=\frac{1}{48}\left(-3 b_{1}^{2} m a_{k}+3 b_{1} m a_{k}^{2}-3 b_{1}^{2} a_{k}+3 b_{1} a_{k}^{2}-m a_{k}^{3}+21 a_{1}^{3}(k-1)+\right. \\
9 a_{2} a_{1}^{2}(k-1)-9 a_{2}^{2} a_{1}(k-1)+3 a_{2}^{3}(k-1)- \\
3 a_{k-1}^{3}-14 a_{k}^{3}-9 a_{k-1} a_{k}^{2}+24 a_{k}^{2}+9 a_{k-1}^{2} a_{k}-12 a_{k}+ \\
\left.b_{2}^{3} m-3 b_{1} b_{2}^{2} m+3 b_{1}^{2} b_{2} m+b_{1}^{3}+2\right) .
\end{gathered}
$$




\section{MRINAL KANTI ROYCHOWDHURY — WASIELA SALINAS}

P r o of. By Lemma 4.9, the optimal set $\alpha_{n}$ of $n$-means contains $k$ elements from $J_{1}$, and $m$ elements from $J_{2}$, where $k=a(n)$ and $m=n-k$. Let $\alpha_{n}:=\left\{a_{1}<a_{2}<\cdots<a_{k}<b_{1}<b_{2}<\cdots<b_{m}\right\}$. Recall Theorem 1.2, and the fact that $P_{1}$ is a uniform distribution on $\left[0, \frac{1}{2}\right]$, and $P_{2}$ is a uniform distribution on $\left[\frac{1}{2}, 1\right]$. Thus, we have

and

$$
a_{j}=\left\{\begin{array}{clc}
\frac{a_{1}+a_{2}}{4} & \text { if } & j=1, \\
\frac{1}{2}\left(\frac{a_{j-1}+a_{j}}{2}+\frac{a_{j}+a_{j+1}}{2}\right) & \text { if } & 2 \leq j \leq k-1,
\end{array}\right.
$$

$$
b_{j}=\left\{\begin{array}{clrl}
\frac{1}{2}\left(\frac{b_{j-1}+b_{j}}{2}+\frac{b_{j}+b_{j+1}}{2}\right) & \text { if } & 2 \leq j \leq m-1, \\
\frac{1}{2}\left(\frac{b_{m-1}+b_{m}}{2}+1\right) & \text { if } & j & =m .
\end{array}\right.
$$

The following two cases can arise:

Case 1. $\frac{1}{2} \leq \frac{1}{2}\left(a_{k}+b_{1}\right)$.

In this case, we have

$$
a_{k}=E\left(X: X \in\left[\frac{a_{k-1}+a_{k}}{2}, \frac{1}{2}\right] \cup\left[\frac{1}{2}, \frac{a_{k}+b_{1}}{2}\right]\right) \text {, and } b_{1}=\frac{1}{2}\left(\frac{a_{k}+b_{1}}{2}+\frac{b_{1}+b_{2}}{2}\right) \text {. }
$$

Case 2. $\frac{1}{2}\left(a_{k}+b_{1}\right)<\frac{1}{2}$.

In this case, we have

$$
a_{k}=\frac{1}{2}\left(\frac{a_{k-1}+a_{k}}{2}+\frac{a_{k}+b_{1}}{2}\right) \text {, and } b_{1}=E\left(X: X \in\left[\frac{a_{k}+b_{1}}{2}, \frac{1}{2}\right] \cup\left[\frac{1}{2}, \frac{b_{1}+b_{2}}{2}\right]\right) \text {. }
$$

For any given positive integer, using the similar techniques as in the proofs of Claim 4.7 and Claim 4.8, we see that in Case 2, the system of equations to obtain $a_{1}, a_{2}, \ldots, a_{k}, b_{1}, \ldots, b_{m}$ does not have any solution. Hence Case 2 cannot happen.

Thus, we have $\frac{1}{2} \leq \frac{1}{2}\left(a_{k}+b_{1}\right)$, i.e., the system of equations to obtain the elements $a_{1}, a_{2}, \ldots, a_{k}, b_{1}, \ldots, b_{m}$ as stated in the theorem are true, and hence, the corresponding quantization error is given by

$$
\begin{aligned}
V_{n}= & \frac{3(k-1)}{4} \int_{0}^{\frac{a_{1}+a_{2}}{2}} 2\left(x-a_{1}\right)^{2} \mathrm{~d} x+\frac{3}{4} \int_{\frac{a_{k-1}+a_{k}}{2}}^{\frac{1}{2}} 2\left(x-a_{k}\right)^{2} \mathrm{~d} x \\
& +\frac{1}{4} \int_{\frac{1}{2}}^{\frac{a_{k}+b_{1}}{2}} 2\left(x-a_{k}\right)^{2} \mathrm{~d} x \frac{m}{4} \int_{\frac{a_{k}+b_{1}}{2}}^{\frac{b_{1}+b_{2}}{2}} 2\left(x-b_{1}\right)^{2} \mathrm{~d} x \\
= & \frac{1}{48}\left(-3 b_{1}^{2} m a_{k}+3 b_{1} m a_{k}^{2}-3 b_{1}^{2} a_{k}+3 b_{1} a_{k}^{2}-m a_{k}^{3}+21 a_{1}^{3}(k-1)\right. \\
& +9 a_{2} a_{1}^{2}(k-1)-9 a_{2}^{2} a_{1}(k-1)+3 a_{2}^{3}(k-1)-3 a_{k-1}^{3}-14 a_{k}^{3}-9 a_{k-1} a_{k}^{2} \\
& \left.+24 a_{k}^{2}+9 a_{k-1}^{2} a_{k}-12 a_{k}+b_{2}^{3} m-3 b_{1} b_{2}^{2} m+3 b_{1}^{2} b_{2} m+b_{1}^{3}+2\right) .
\end{aligned}
$$




\section{QUANTIZATION FOR A MIXTURE OF UNIFORM DISTRIBUTIONS}

Thus, we complete the proof of the theorem.

Now, we give the following example.

EXAmple 4.11. Take $n=16$. Then, $k=a(n)=10$, and so, $m=6$. Thus, by Theorem 4.10, we have

$$
\left.\begin{array}{rlll}
\left\{a_{1}=0.0255733,\right. & a_{2}=0.0767199, & a_{3}=0.127866, & a_{4}=0.179013 \\
a_{5}=0.23016, & a_{6}=0.281306, & a_{7}=0.332453, & a_{8}=0.383599 \\
a_{9}=0.434746, & a_{10}=0.485893, & b_{1}=0.564986, & b_{2}=0.644079 \\
b_{3}=0.723173, & b_{4}=0.802266, & b_{5}=0.88136, & b_{6}=0.960453,
\end{array}\right\}
$$

and the corresponding quantization error is given by

$$
\begin{gathered}
V_{16}=\frac{1}{48}\left(-21 a_{10} b_{1}^{2}+21 a_{10}^{2} b_{1}+189 a_{1}^{3}+81 a_{2} a_{1}^{2}-81 a_{2}^{2} a_{1}+\right. \\
27 a_{2}^{3}-3 a_{9}^{3}-20 a_{10}^{3}-9 a_{9} a_{10}^{2}+24 a_{10}^{2}+9 a_{9}^{2} a_{10}- \\
\left.12 a_{10}+b_{1}^{3}+6 b_{2}^{3}-18 b_{1} b_{2}^{2}+18 b_{1}^{2} b_{2}+2\right)=0.000293827 .
\end{gathered}
$$

\section{REFERENCES}

[BW] BUCKLEW, J.A.- WISE, G.L.: Multidimensional asymptotic quantization theory with rth power distortion measures, IEEE Trans. Inform. Theory, 28 (1982), no. 2, 239-247.

[CR] ÇÖMEZ, D.-ROYCHOWDHURY, M.K.: Quantization for uniform distributions on stretched Sierpiński triangles, Monatsh.Math. 190 (2019), no. 1, 79-100.

[DR1] DETTMANN, C.P.-ROYCHOWDHURY, M. K.: Quantization for uniform distributions on equilateral triangles, Real Anal. Exchange, 42 (2017), no. (1), 149-166.

[DR2] DETTMANN, C.P.-ROYCHOWDHURY, M.K.: An algorithm to compute CVTs for finitely generated Cantor distributions, Southeast Asian Bull. Math. (to appear).

[GG] GERSHO, A.-GRAY, R. M.: Vector Quantization and Signal Compression. Kluwer Academy publishers: Boston, MA, 1992.

[GL1] GRAF, S.-LUSCHGY, H.: Foundations of Quantization for Probability Distributions. In: Lecture Notes in Mathematics Vol. 1730, Springer-Verlag, Berlin, 2000.

[GL2] GRAF, S.-LUSCHGY, H.: The Quantization of the Cantor distribution, Math. Nachr. 183 (1997), 113-133.

[GN] GRAY, R. M.-NEUHOFF, D. L.: Quantization. IEEE Trans. Inform. Theory, 44 (1998), 2325-2383.

[L] LINDSAY, L.J.: Quantization dimension for probability distributions, Thesis (Ph.D.)-University of North Texas. ProQuest LLC, Ann Arbor, MI, 2001. 


\section{MRINAL KANTI ROYCHOWDHURY — WASIELA SALINAS}

[L1] ROYCHOWDHURY, L.: Optimal quantization for nonuniform Cantor distributions, J. Interdiscip. Math. 22 (2019), no. 8, 1325-1348.

[P] PÖTZELBERGER, K.: The quantization dimension of distributions, Math. Proc. Cambridge Philos. Soc. 131 (2001), no. 3, 507-519.

[R1] ROYCHOWDHURY, M.K.: Quantization and centroidal Voronoi tessellations for probability measures on dyadic Cantor sets, J. Fractal Geom. 4 (2017), no. 2, 127-146.

[R2] ROYCHOWDHURY, M. K.: Optimal quantizers for some absolutely continuous probability measures, Real Anal. Exchange 43 (2017), no. 1, 105-136.

[R3] ROYCHOWDHURY, M. K.: Optimal quantization for the Cantor distribution generated by infinite similitudes, Israel J. Math. 231 (2019), no. 1, 437-466.

[R4] ROYCHOWDHURY, M. K.: Least upper bound of the exact formula for optimal quantization of some uniform Cantor distributions, Discrete Contin. Dyn. Syst. Ser. A, 38 (2018), no. 9, 4555-4570.

[R5] ROYCHOWDHURY, M. K.: Center of mass and the optimal quantizers for some continuous and discrete uniform distributions, J. Interdiscip. Math. 22 (2019), no. 4, 451-471.

[RR1] ROSENBLATT, J.-ROYCHOWDHURY, M. K.: Optimal quantization for piecewise uniform distributions, Unif. Distrib. Theory 13 (2018), no. 2, 23-55.

[RR2] ROSENBLATT, J.-ROYCHOWDHURY, M. K.: Uniform distributions on curves and quantization, arXiv:1809.08364 [math.PR].

[Z] ZAMIR, R.: Lattice Coding for Signals and Networks: A Structured Coding Approach to Quantization, Modulation, and Multiuser Information Theory. Cambridge University Press, Cambridge, 2014.

Received October 18, 2019

Accepted March 1, 2020

\section{Mrinal Kanti Roychowdhury Wasiela Salinas}

School of Mathematical and

Statistical Sciences

University of Texas Rio Grande Valley

1201 West University Drive

Edinburg, TX 78539-2999, USA.

E-mail: mrinal.roychowdhury@utrgv.edu wsalinas47@gmail.com 